\title{
A nonlocal multiscale discrete-continuum model for predicting mechanical behavior of granular materials
}

\author{
Yang Liu, WaiChing Sun*, ${ }^{*}$ Zifeng Yuan and Jacob Fish \\ Department of Civil Engineering and Engineering Mechanics, Columbia University, New York, 10027, NY, USA
}

\begin{abstract}
SUMMARY
A three-dimensional nonlocal multiscale discrete-continuum model has been developed for modeling mechanical behavior of granular materials. In the proposed multiscale scheme, we establish an informationpassing coupling between the discrete element method, which explicitly replicates granular motion of individual particles, and a finite element continuum model, which captures nonlocal overall responses of the granular assemblies. The resulting multiscale discrete-continuum coupling method retains the simplicity and efficiency of a continuum-based finite element model, while circumventing mesh pathology in the postbifurcation regime by means of staggered nonlocal operator. We demonstrate that the multiscale coupling scheme is able to capture the plastic dilatancy and pressure-sensitive frictional responses commonly observed inside dilatant shear bands, without employing a phenomenological plasticity model at a macroscopic level. In addition, internal variables, such as plastic dilatancy and plastic flow direction, are now inferred directly from granular physics, without introducing unnecessary empirical relations and phenomenology. The simple shear and the biaxial compression tests are used to analyze the onset and evolution of shear bands in granular materials and sensitivity to mesh density. The robustness and the accuracy of the proposed multiscale model are verified in comparisons with single-scale benchmark discrete element method simulations. Copyright (C) 2015 John Wiley \& Sons, Ltd.
\end{abstract}

Received 24 November 2014; Revised 26 September 2015; Accepted 28 September 2015

KEY WORDS: multiscale discrete-continuum model; staggered nonlocal operator; strain localization; granular materials; homogenization; shear band; anisotropy

\section{INTRODUCTION}

While the macroscopic response of granular materials may appear to be similar to those of continua, it essentially represents a collective behavior of interacting particles. For example, the rearrangement and crushing of particles, collapse of void space, buckling and splitting of force chains, may result in path-dependent responses of granular materials at macroscale, such as plastic dilatancy, nonassociative plastic flow and strain localization.

Over the last three decades, computer simulations of granular motion have gained increasing attention. Several classes of models have been proposed to replicate the behavior of the granular media including:

(i) discrete approaches that explicitly model the particulate interactions among particle contacts at the grain scale

(ii) continuum approaches that characterize path-dependent responses with internal variables and constitutive laws at macroscopic scales, and

(iii) multiscale approaches that concurrently or by means of information-passing that link both (i) and (ii).

\footnotetext{
*Correspondence to: WaiChing Sun, Assistant Professor, Department of Civil Engineering \& Engineering Mechanics, Columbia University, 614 Seeley W. Mudd, 500 West 120th Street, New York, NY 10027, USA.

†E-mail: wsun@columbia.edu 
The continuum approach has been widely used in mining, petroleum, and geotechnical engineering problems to approximate the collective behavior of granular media at the field scale. Phenomenological plasticity models that successfully resolve macroscopic behavior of specific types of granular materials such as sand, silt, and powder have been extensively reported in the literature $[1-10]$. However, if no length scale is introduced in the phenomenological plasticity models, spurious mesh dependence may still occur at the post-bifurcation regime. Furthermore, phenomenological model relies on the usage of internal variables to replicate path-dependent behaviors. It remains a difficult task to directly link or even replicate all different dissipation mechanisms originating from the grain scales, such as granular vertex and force chain buckling [11-13], by the evolution of internal variables alone.

The discrete element method (DEM) provides a simple but computationally intensive solution to resolve the aforementioned deficiencies of continuum approaches for granular materials. In DEM, motion of grains is explicitly resolved based on contacts and long-range interactive mechanisms among particles [14-19] Nevertheless, because DEM explicitly models and tracks the motion of each individual particle in the grain assembly, the computational cost is often too high for practical engineering problems that are in large spatial and time scales.

To overcome this issue, various concurrent and information-passing multiscale methods have been proposed to couple grain-scale simulations with macroscopic continuum-scale finite element analyses [20-29]. For instance, Wellmann and Wriggers [20] introduced an Arlequin DEM-FEM model that divides the spatial domain into discrete and continuum subdomains. Parts of the discrete and continuum subdomains are overlapped with each other to create a handshake region such that spurious reflection can be suppressed. Li and Wan [21] and Regueiro and Yan [22] proposed bridging scale method, which uses a handshake domain to couple particulate model with higher-order continua.

The homogenization-based multiscale discrete-continuum coupling technique is pioneered by Miehe and Dettmar $[23,24]$ in which a micro-macro transition is established by locally attaching microstructures with macro-continuum at finite strain. Macroscopic stress tensor is then obtained from the DEM by deforming a periodic Lagrangian frame that contains the granular microstructures. Miehe et al. [23, 24] extended Hill-Mandel microhomogeneity condition from continuous heterogeneous systems to granular materials. These studies reveal that the responses obtained via the linear displacement and uniform stress boundary conditions represent the upper and lower bounds of the stiffness, while the periodic boundary condition is the optimal choice at which coarse-scale properties converges faster with respect to the representative volume element (RVE) size.

Stránský and Jirásek [25], Nguyen et al. [26] and Guo and Zhao [27] proposed a conceptually similar approach where homogenized stress measures and the tangent operator inferred from periodic discrete element simulations conducted on a RVE are directly used to update an otherwise conventional small strain implicit finite element model [30-33]. Andrade and Tu [28] proposed a staggered multiscale constitutive model in which evolutions of the yield surfaces and plastic potential are governed by DEM simulations or meso-scale experiments. This multiscale constitutive model is then used to update the Cauchy stress and consistent tangent operator of an implicit small strain finite element model. These information-passing DEM-FEM coupling approaches have proven to be stable. Nevertheless, both Nguyen et al. [26] and Guo and Zhao [27] concluded that the implicit DEM-FEM coupling model suffers two drawbacks - (i) a large number of DEM iteration steps is required to reach local convergence, and (ii) the post-bifurcation responses obtained from such an information-passing coupling model may exhibit strong mesh dependence. Furthermore, while the computational cost of the information-passing DEM-FEM coupling model is substantially lower than of a single-scale DEM, the Newton-Raphson scheme used to update the finite element solution often requires multiple DEM simulations to achieve convergences. This can be a significant issue in the post softening regime where stress-strain curves obtained from DEM are typically bumpy and sensitive to perturbations. According to Guo and Zhao [27], the information-passing multiscale scheme may require as much as 48 DEM simulations for each quadrature point. Except [23, 24], the aforementioned hierarchical DEM-FEM coupling methods are all formulated in the geometrically linear regime and thus may not be suitable for shear-banding problem where significant plastic spin may develop [34]. 
In the present manuscript, we develop a nonlocal multiscale discrete-continuum model based on the Generalized Mathematical Homogenization (GMH) originally developed for linking atomisticcontinuum scales [30-33]. GMH belongs to the category of information-passing multiscale methods, which evolve a coarse-scale model by advancing a sequence of fine-scale models in small windows (or RVEs) placed at the quadrature points of the finite element model. Consequently, GMH gives rise to constitutive law-free coarse-scale equations where the coarse-scale continuum model is directly driven by discrete element simulations at the grain scales. The primary goal of the present work is to develop an information-passing DEM-FEM coupling scheme that (i) satisfactorily resolves both the overall and fine-scale responses of the granular media, (ii) is computationally efficient, and (iii) overcomes pathological mesh sensitivity in the post-bifurcation regime. The contributions of the present work are summarized as follows:

(i) Alleviating mesh sensitivity in post-bifurcation regime. Previous hierarchical DEM-FEM coupling schemes have proven to be mesh dependence in $[26,27]$ after the onset of strain localization. The proposed multiscale approach remedies this issue by applying a modified staggered nonlocal approach proposed in $[35,36]$ to define the unit cell problem for the stress homogenization.

(ii) Formulating the two-scale discrete-continuum problem via the GMH framework. This treatment allows us to derive the Cauchy stress expression directly from the equilibrium equations of particles and provide a consistent framework that links the continuum (coarse-scale) and discrete (fine-scale) representations of the granular assemblies based on the multiscale asymptotic analysis. We also establish the connection between the GMH and the Hill-Mandel condition and prove that the latter is a specific case of GMH in which coarse and fine scales are in the same temporal scales [37-39].

The rest of the paper is organized as follows. In Section 2, the governing equations at a scale of particles are briefly reviewed. The theoretical background established via GMH to obtain constitutive-law free coarse-scale equations is then described, followed by the computational aspects of the proposed nonlocal multiscale scheme. Numerical examples, including a cyclic simple shear test, the monotonic simple shear test, and the biaxial compression test, are presented in Section 3 to verify the model against a single-scale DEM simulation. Observations and conclusions are presented in Section 4.

\section{METHOD}

In this section, we provide the theoretical basis for the nonlocal multiscale scheme that couples the grain-scale discrete mechanics simulations and the macroscopic continuum model via a modified version of GMH, as depicted in Figure 1. We first formulate the micro-macro transition for the granular assemblies via a multiscale asymptotic analysis. This treatment allows one to associate the macroscopic quadrature point with unit cell consisting of particles. We then provide a brief description of the coarse-scale finite element model that replicates the continuum scale behaviors, and the unit cell discrete element method that replaces the macroscopic phenomenological internal variables to provide incremental constitutive update to the macroscopic problem. Because of the usage of the conditionally stable explicit scheme, we analyze the relations of the coarse-scale and fine-scale critical time steps. The staggered scheme used to integrate the nonlocal quadrature is also discussed.

\subsection{Micro-macro transition for granular assemblies via asymptotic expansion}

We consider a unit cell consisting of $n$ particles. With the interior domain of the unit cell, these particles may exert contact force and torque on their neighboring particles. The initial position of particle $I$ is denoted as $\mathbf{X}^{I}$, and its displacement is denoted as $\mathbf{u}^{I}$. Thus, the current position of the Ith particle is as follows:

$$
\mathbf{x}^{I}=\mathbf{X}^{I}+\mathbf{u}^{I}
$$




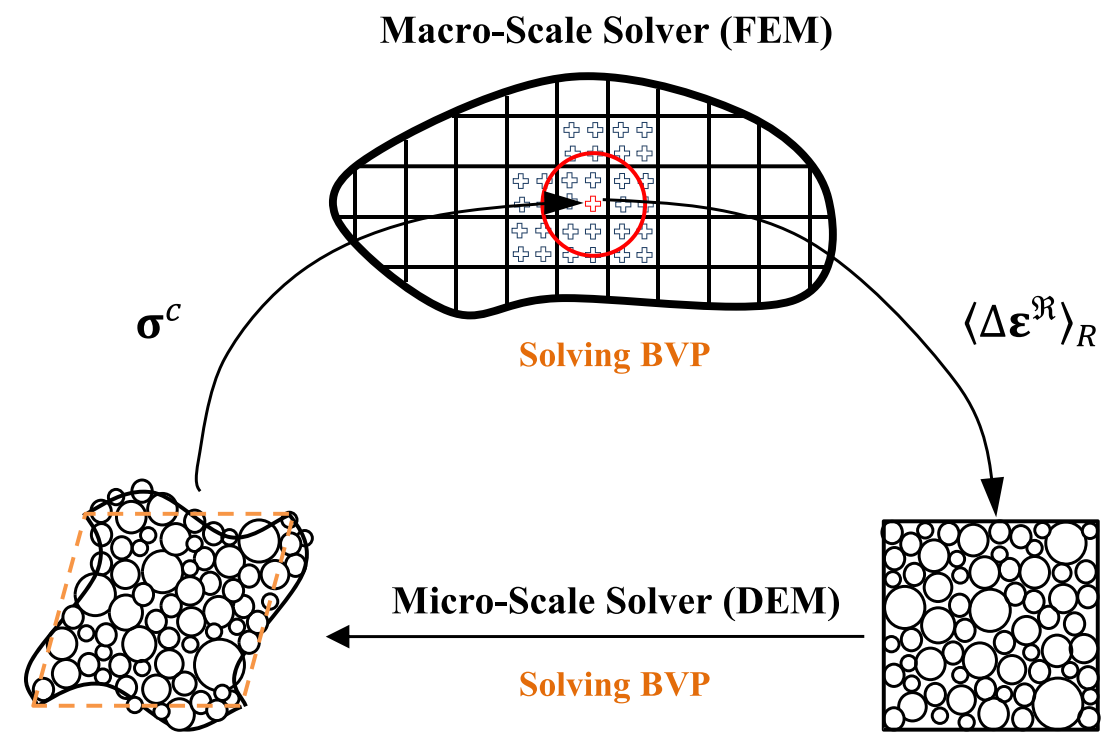

Figure 1. Information flow in the nonlocal two-scale discrete-continuum model. $\left\langle\Delta \varepsilon^{\Re}\right\rangle_{R}$ is the nonlocal coarse-scale corotational strain increment; $\sigma^{c}$ is the coarse-scale Cauchy stress.

The distance of two particles $I$ and $J$ in the initial configuration is as follows:

$$
\mathbf{X}^{I J}=\mathbf{X}^{J}-\mathbf{X}^{I}
$$

and in the current configuration, the distance is as follows:

$$
\mathbf{x}^{I J}=\mathbf{x}^{J}-\mathbf{x}^{I}=\mathbf{X}^{I J}+\mathbf{u}^{J}\left(\mathbf{X}^{J}, t\right)-\mathbf{u}^{I}\left(\mathbf{X}^{I}, t\right)
$$

Given the interaction between particles $I$ and $J$, the equations of motion for particle $I$ can thus be expressed by the following:

$$
m^{I} \ddot{\mathbf{u}}^{I}=\sum_{J \neq I} \mathbf{f}^{I J}\left(\mathbf{x}^{I J}\right)+\mathbf{F}_{\text {ext }}^{I}
$$

where $m^{I}$ is the mass of particle $I ; \ddot{\mathbf{u}}^{I}$ and $\dot{\mathbf{u}}^{I}$ are the acceleration and velocity of particle center of mass, respectively. $\mathbf{f}^{I J}$ is the internal contact force applied to particle $I$ by particle $J ; \mathbf{F}_{\text {ext }}^{I}$ is resultant external force applied to particle $I$, such as boundary force or body force. The superscript $J$ denotes the neighboring particles that interact with particle $I$, such that $\left|\mathbf{x}^{J}-\mathbf{x}^{I}\right|<r_{c}$, with $r_{c}$ being the cutoff radius. The mass of the I-th particle $m^{I}$, interval force $\mathbf{f}^{I J}$, and external force $\mathbf{F}_{\text {ext }}^{I}$ are assumed to be periodic functions because of local periodicity of the grain assembly.

A particle moves against its neighboring particles by indenting, sliding and/or rolling at contact points. While the dominant role of sliding was considered in classical theories of strength and dilatancy of granular materials [40], previous research, such as Oda and Iwashita [41], suggests that rolling, rather than sliding, is a dominant micro-deformation mechanism leading to extensive dilatancy of granular media . Mühlhaus and Vardoulakis [42] conducted a bifurcation analysis based on a micropolar theory and successfully predicted the thickness of a shear band, as well as the shear band direction. Brown and Evans [43] questioned the need to incorporate micropolar terms for granular media, based on the fact that the coupled stress might be extremely small in most circumstances. In this paper, we do not incorporate the rolling and torsional resistances. Because rotational stiffness is not introduced in our DEM model, higher-order kinematic measures, such as particles rotation gradient, are not incorporated in the homogenized responses. The homogenized Cauchy stress tensor components are assumed to be symmetric, and no couple stress is used to formulate a complete set of the governing equations. 
In the multiscale discrete-continuum method, two distinct spatial coordinates are employed to describe the heterogeneity at a grain level as follows: (i) the coarse-scale coordinate, denoted by $\mathbf{X}$, in the coarse-scale domain $\boldsymbol{\Omega}$, at which the grain scale features are invisible, and (ii) the grain-scale or fine-scale coordinate, denoted by $\mathbf{Y}$, in the discrete unit cell domain $\boldsymbol{\Theta}$. Assuming that the dimension of heterogeneity is significantly smaller than the characteristic size of the macroscopic problem, the macro-scale and micro-scale coordinate systems of the reference configuration are related by:

$$
\mathbf{Y}=\mathbf{X} / \xi
$$

where $\xi$ is a small positive scaling parameter that $0<\xi \ll 1$. The corresponding spatial scales in the current configuration are denoted by $\mathbf{x}$ and $\mathbf{y}$, respectively, and are related by $\mathbf{y}=\mathbf{x} / \xi$. We assume that the coarse-scale coordinate $\mathbf{X}$ takes continuous series of values, and displacements $\mathbf{u}(\mathbf{X}, \mathbf{Y}, t)$ are continuous and differentiable in $\mathbf{X}$, while the fine-scale coordinate $\mathbf{Y}$ is discrete.

We follow the derivation steps in [30-33] to derive the two-scale formation. However, unlike atomistic simulations that involve multiple time scales due to atomistic vibrations, a single time scale is considered. The first two material time derivatives of the displacement field are given by:

$$
\frac{d \mathbf{u}\left(\mathbf{X}, \mathbf{Y}^{I}, t\right)}{d t}=\dot{\mathbf{u}}^{I} \quad \frac{d^{2} \mathbf{u}\left(\mathbf{X}, \mathbf{Y}^{I}, t\right)}{d t^{2}}=\ddot{\mathbf{u}}^{I} .
$$

Prior to carrying out the multiple scale asymptotic analysis, it is necessary to rescale Equation (4). We start by considering continuum equations of motion $\rho_{0} \ddot{\mathbf{u}}(\mathbf{X}, t)-\nabla_{\mathbf{X}} \cdot \mathbf{P}=0$ where $\rho_{0}$ is the mass density; $\mathbf{P}$ is the first Piola-Kirchhoff stress tensor and $\nabla_{\mathbf{X}} \cdot \mathbf{P}$ denotes the divergence of stress tensor $\mathbf{P}$. For homogeneous media, stress derivatives are of order one, whereas for heterogeneous media, for which certain components of stresses are discontinuous, stress derivatives are of $O\left(\xi^{-1}\right)$. Assuming that the material density is $\rho_{0} \sim O(1)$ and the characteristic size of the unit cell is $l \sim O(\xi)$, the volume of the unit cell is $\Theta_{0} \sim O\left(\xi^{3}\right)$. Thus, the mass $m \sim \rho_{0} \Theta_{0} \sim O\left(\xi^{3}\right)$. Dividing Equation (4) by volume of the unit cell yields the following:

$$
k_{1} \rho_{0} \ddot{\mathbf{u}}^{I}=\frac{1}{k_{2} \xi^{3}} \sum_{J \neq I} \mathbf{f}^{I J}\left(\mathbf{x}^{I J}\right)+\frac{1}{k_{3} \xi^{3}} \mathbf{F}_{\mathrm{ext}}^{I}
$$

where $k_{1}, k_{2}$ and $k_{3}$ are order one constants. Comparing Equation (7) to the continuum equations of motion, we obtain the following:

$$
\mathbf{f}^{I J}\left(\mathbf{x}^{I J}\right) \sim O\left(\xi^{2}\right), \quad \mathbf{F}_{\mathrm{ext}} \sim O\left(\xi^{2}\right) .
$$

Then we introduce the following $O(1)$ normalized quantities

$$
\bar{m}=m / \xi^{3} \sim O(1) ; \overline{\mathbf{f}}^{I J}=\mathbf{f}^{I J} / \xi^{2} \sim O(1) ; \overline{\mathbf{F}}_{\mathrm{ext}}=\mathbf{F}_{\mathrm{ext}} / \xi^{2} \sim O(1) .
$$

Therefore, Equation (4) can be rewritten as:

$$
\bar{m} \ddot{\mathbf{u}}^{I}=\frac{1}{\xi} \sum_{J \neq I} \overline{\mathbf{f}}^{I J}+\frac{1}{\xi} \overline{\mathbf{F}}_{\mathrm{ext}}
$$

A multiscale asymptotic expansion is employed to approximate the displacement field as:

$$
\mathbf{u}(\mathbf{X}, \mathbf{Y}, t)=\mathbf{u}^{(0)}(\mathbf{X}, t)+\xi \mathbf{u}^{(1)}(\mathbf{X}, \mathbf{Y}, t)+\ldots
$$

where the leading order displacement $\mathbf{u}^{(0)}$ is termed the coarse-scale displacement, $\mathbf{u}^{c} \equiv \mathbf{u}^{(0)}$. It is assumed to be independent of the fine-scale coordinate. Inserting Equation (11) into Equation (6) yields the following: 


$$
\begin{aligned}
\dot{\mathbf{u}}^{I} & =\dot{\mathbf{u}}^{c}+O(\xi) \\
\ddot{\mathbf{u}}^{I} & =\ddot{\mathbf{u}}^{c}+O(\xi) .
\end{aligned}
$$

We denote the displacement of the I-th particle by $u_{i}^{I}=u_{i}\left(\mathbf{X}, \mathbf{Y}^{I}, t\right)$ with $\mathbf{X}=\mathbf{X}^{I}$. The displacements of the neighboring particle $u_{i}^{J}\left(\mathbf{X}^{J}, \mathbf{Y}^{J}, t\right)$ can be expanded using a Taylor series around point $\mathbf{X}$ as follows:

$$
u_{i}^{J}=u_{i}\left(\mathbf{X}, \mathbf{Y}^{J}, t\right)+\frac{\partial u_{i}\left(\mathbf{X}, \mathbf{Y}^{J}, t\right)}{\partial X_{j}} X_{j}^{I J}+\frac{1}{2} \frac{\partial^{2} u_{i}\left(\mathbf{X}, \mathbf{Y}^{J}, t\right)}{\partial X_{j} \partial X_{k}} X_{j}^{I J} X_{k}^{I J}+\ldots
$$

From Equation (13), we have:

$$
\begin{aligned}
u_{i}^{J}-u_{i}^{I}= & u_{i}\left(\mathbf{X}, \mathbf{Y}^{J}, t\right)-u_{i}\left(\mathbf{X}, \mathbf{Y}^{I}, t\right)+\frac{\partial u_{i}\left(\mathbf{X}, \mathbf{Y}^{J}, t\right)}{\partial X_{j}} X_{j}^{I J}+ \\
& +\frac{1}{2} \frac{\partial^{2} u_{i}\left(\mathbf{X}, \mathbf{Y}^{J}, t\right)}{\partial X_{j} \partial X_{k}} X_{j}^{I J} X_{k}^{I J}+\ldots
\end{aligned}
$$

Inserting Equation (5) into Equation (14) yields

$$
\begin{aligned}
u_{i}^{J}-u_{i}^{I}=u_{i} & \left(\mathbf{X}, \mathbf{Y}^{J}, t\right)-u_{i}\left(\mathbf{X}, \mathbf{Y}^{I}, t\right)+\xi \frac{\partial u_{i}\left(\mathbf{X}, \mathbf{Y}^{J}, t\right)}{\partial X_{j}} Y_{j}^{I J} \\
& +\frac{1}{2} \xi^{2} \frac{\partial^{2} u_{i}\left(\mathbf{X}, \mathbf{Y}^{J}, t\right)}{\partial X_{j} \partial X_{k}} Y_{j}^{I J} Y_{k}^{I J}+\ldots
\end{aligned}
$$

Inserting the asymptotic expansion Equation (11) into Equation (15) yields:

$$
\begin{aligned}
u_{i}^{J}-u_{i}^{I}= & \xi\left(u_{i}^{(1)}\left(\mathbf{X}, \mathbf{Y}^{J}, t\right)-u_{i}^{(1)}\left(\mathbf{X}, \mathbf{Y}^{I}, t\right)+\frac{\partial u_{i}^{c}(\mathbf{X}, t)}{\partial X_{j}} Y_{j}^{I J}\right) \\
& +\frac{1}{2} \xi^{2}\left(\frac{\partial u_{i}^{(1)}\left(\mathbf{X}, \mathbf{Y}^{J}, t\right)}{\partial X_{j}} Y_{j}^{I J}+\frac{1}{2} \frac{\partial^{2} u_{i}^{c}(\mathbf{X}, t)}{\partial X_{j} \partial X_{k}} Y_{j}^{I J} Y_{k}^{I J}\right)+\ldots
\end{aligned}
$$

Inserting Equation (16) into Equation (3) yields the following:

$$
\begin{aligned}
& \mathbf{x}^{I J}=\mathbf{X}^{I J}+\mathbf{u}^{J}-\mathbf{u}^{I}=\xi \boldsymbol{\varphi}^{I J}+\xi^{2} \boldsymbol{\Psi}^{I J}+\ldots \\
& \mathbf{y}^{I J}=\mathbf{x}^{I J} / \xi=\boldsymbol{\varphi}^{I J}+\xi \boldsymbol{\Psi}^{I J}+\ldots
\end{aligned}
$$

where

$$
\begin{aligned}
& \varphi_{i}^{I J}=F_{i j}^{c}(\mathbf{X}) Y_{j}^{I J}+u_{i}^{(1)}\left(\mathbf{X}, \mathbf{Y}^{J}, t\right)-u_{i}^{(1)}\left(\mathbf{X}, \mathbf{Y}^{I}, t\right) \\
& \Psi_{i}^{I J}=\frac{\partial u_{i}^{(1)}\left(\mathbf{X}, \mathbf{Y}^{J}, t\right)}{\partial X_{j}} Y_{j}^{I J}+\frac{1}{2} \frac{\partial^{2} u_{i}^{c}(\mathbf{X})}{\partial X_{j} \partial X_{k}} Y_{j}^{I J} Y_{k}^{I J} .
\end{aligned}
$$

Herein, $F_{i j}^{c}(\mathbf{X}, t)$ denotes the coarse-scale deformation gradient, i.e.,

$$
F_{i j}^{c}(\mathbf{X})=\delta_{i j}+\frac{\partial u_{i}^{c}(\mathbf{X})}{\partial X_{j}} .
$$

The contact force $\mathbf{f}^{I J}$ is a function of $\mathbf{x}^{I J}$ so that

$$
\begin{aligned}
\bar{f}_{i}^{I J} & =\bar{f}_{i}^{I J}\left(\xi \mathbf{y}^{I J}\right)=\bar{f}_{i}^{I J}\left(\xi \boldsymbol{\varphi}^{I J}+\xi^{2} \boldsymbol{\Psi}^{I J}+\ldots\right) \\
& =\bar{f}_{i}^{I J}\left(\xi \boldsymbol{\varphi}^{I J}\right)+\left.\frac{\partial \bar{f}_{i}^{I J}}{\partial y_{k}^{I J}} \frac{\partial y_{k}^{I J}}{\partial x_{j}^{I J}}\right|_{\mathbf{y}^{I J}=\boldsymbol{\varphi}^{I J}} \xi^{2} \Psi_{j}^{I J}+O\left(\xi^{2}\right) \\
& =\hat{\bar{f}}_{i}^{I J}+\left.\xi \frac{\partial \bar{f}_{i}^{I J}}{\partial y_{j}^{I I}}\right|_{\mathbf{y}^{I J}=\boldsymbol{\varphi}^{I J}} \Psi_{j}^{I J}+O\left(\xi^{2}\right)
\end{aligned}
$$


where

$$
\hat{\bar{f}}_{i}^{I J}=\hat{\bar{f}}_{i}^{I J}\left(\xi \boldsymbol{\varphi}^{I J}\right)
$$

Inserting Equations (12) and (20) into Equation (10) yields the following:

$$
\bar{m}^{I} \ddot{u}_{i}^{c}(\mathbf{X}, t)=\frac{1}{\xi} \sum_{J \neq I}\left(\hat{\bar{f}}_{i}^{I J}+\left.\xi \frac{\partial \bar{f}_{i}^{I J}}{\partial y_{j}^{I J}}\right|_{\mathbf{y}^{I J}=\boldsymbol{\varphi}^{I J}} \Psi_{j}^{I J}\right)+\frac{1}{\xi} \bar{F}_{e x t, i}\left(\mathbf{u}^{I}\right) .
$$

Collecting terms of equal power of $\xi$ gives the equations of motion at different scales

$$
\begin{gathered}
O\left(\xi^{-1}\right): \quad \sum_{J} \hat{f}_{i}^{I J}+\bar{F}_{e x t, i}\left(\mathbf{u}^{I}\right)=0 \\
O(1): \quad m \ddot{u}_{i}^{c}(\mathbf{X}, t)=\sum_{J}\left(\left.\frac{\partial f_{i}^{I J}}{\partial y_{j}^{I J}}\right|_{\mathbf{y}^{I J}=\boldsymbol{\varphi}^{I J}} \Psi_{j}^{I J}\right) .
\end{gathered}
$$

Equation (23) is a quasi-static unit cell problem. We now focus on the coarse-scale problem.

Summation over all the particles and then dividing Equation (24) by the volume of the unit cell $\left|\Theta_{0}\right|$ for the initial configuration give the following:

$$
\frac{1}{\left|\Theta_{0}\right|} \sum_{I} m^{I} \ddot{u}_{i}^{c}(\mathbf{X}, t)=\left.\frac{1}{\left|\Theta_{0}\right|} \sum_{I} \sum_{J} \frac{\partial f_{i}^{I J}}{\partial y_{j}^{I J}}\right|_{\mathbf{y}^{I J}=\boldsymbol{\varphi}^{I J}} \Psi_{j}^{I J} .
$$

It is noted that the density is defined as:

$$
\rho_{0}^{c}=\frac{1}{\left|\Theta_{0}\right|} \sum_{I} m^{I}
$$

so that

$$
\rho_{0}^{c} \ddot{u}_{i}^{c}(\mathbf{X}, t)=\left.\frac{1}{\left|\Theta_{0}\right|} \sum_{I} \sum_{J} \frac{\partial f_{i}^{I J}}{\partial y_{j}^{I J}}\right|_{\mathbf{y}^{I J}=\boldsymbol{\varphi}^{I J}} \Psi_{j}^{I J}
$$

Considering Equations (17) and (18), we have the following:

$$
\begin{aligned}
f_{i, X_{j}}^{I J} & =\frac{\partial f_{i}^{I J}}{\partial y_{k}^{I J}} \frac{\partial y_{k}^{I J}}{\partial X_{j}}=\frac{\partial f_{i}^{I J}}{\partial y_{k}^{I J}} \frac{\partial \varphi_{k}^{I J}}{\partial X_{j}}+O(\xi) \\
& =\frac{\partial f_{i}^{I J}}{\partial y_{k}^{I J}}\left(\frac{\partial^{2} u_{k}^{c}(\mathbf{X}, t)}{\partial X_{m} \partial X_{j}} Y_{m}^{I J}+\frac{\partial u_{k}^{(1)}\left(\mathbf{X}, \mathbf{Y}^{J}, t\right)}{\partial X_{j}}-\frac{\partial u_{k}^{(1)}\left(\mathbf{X}, \mathbf{Y}^{I}, t\right)}{\partial X_{j}}\right)+O(\xi) .
\end{aligned}
$$

In the RHS of Equation (27), we have 


$$
\begin{aligned}
\left.\frac{\partial f_{i}^{I J}}{\partial y_{j}^{I I}}\right|_{\mathbf{y}^{I J}=\boldsymbol{\varphi}^{I J}} \Psi_{j}^{I J}= & \left.\frac{\partial f_{i}^{I J}}{\partial y_{j}^{I J}}\right|_{\mathbf{y}^{I J}=\boldsymbol{\varphi}^{I J}}\left(\frac{\partial u_{j}^{(1)}\left(\mathbf{X}, \mathbf{Y}^{J}, t\right)}{\partial X_{m}}+\frac{1}{2} \frac{\partial^{2} u_{j}^{c}(\mathbf{X})}{\partial X_{m} \partial X_{k}} Y_{k}^{I J}\right) Y_{m}^{I J} \\
= & \left.\frac{1}{2} \frac{\partial f_{i}^{I J}}{\partial y_{j}^{I J}}\right|_{\mathbf{y}^{I J}=\boldsymbol{\varphi}^{I J}}\left(\frac{\partial u_{j}^{(1)}\left(\mathbf{X}, \mathbf{Y}^{J}, t\right)}{\partial X_{m}}-\frac{\partial u_{j}^{(1)}\left(\mathbf{X}, \mathbf{Y}^{I}, t\right)}{\partial X_{m}}+\frac{\partial^{2} u_{j}^{c}(\mathbf{X})}{\partial X_{m} \partial X_{k}} Y_{k}^{I J}\right) Y_{m}^{I J} \\
& \left.+\left.\frac{1}{2} \frac{\partial f_{i}^{I J}}{\partial y_{j}^{I J}}\right|_{\mathbf{y}^{I J}=\boldsymbol{\varphi}^{I J}}\left(\frac{\partial u_{j}^{(1)}\left(\mathbf{X}, \mathbf{Y}^{J}, t\right)}{\partial X_{m}}+\frac{\partial u_{j}^{(1)}\left(\mathbf{X}, \mathbf{Y}^{I}, t\right)}{\partial X_{m}}\right) Y_{m}^{I J}\right) \\
= & \frac{1}{2} \frac{\partial}{\partial X_{j}}\left(f_{i}^{I J} Y_{j}^{I J}\right)+\left.\frac{1}{2} \frac{\partial f_{i}^{I J}}{\partial y_{j}^{I J}}\right|_{\mathbf{y}^{I J}=\boldsymbol{\varphi}^{I J}}\left(\frac{\partial u_{j}^{(1)}\left(\mathbf{X}, \mathbf{Y}^{J}, t\right)}{\partial X_{m}}+\frac{\partial u_{j}^{(1)}\left(\mathbf{X}, \mathbf{Y}^{I}, t\right)}{\partial X_{m}}\right) Y_{m}^{I J} \\
= & \frac{1}{2 \xi} \frac{\partial}{\partial X_{j}}\left(f_{i}^{I J} X_{j}^{I J}\right)+\left.\frac{1}{2} \frac{\partial f_{i}^{I J}}{\partial y_{j}^{I J}}\right|_{\mathbf{y}^{I J}=\boldsymbol{\varphi}_{I J}^{I J}}\left(\frac{\partial u_{j}^{(1)}\left(\mathbf{X}, \mathbf{Y}^{J}, t\right)}{\partial X_{m}}+\frac{\partial u_{j}^{(1)}\left(\mathbf{X}, \mathbf{Y}^{I}, t\right)}{\partial X_{m}}\right) Y_{m}^{I J} .
\end{aligned}
$$

Inserting Equation (29) into Equation (27) yields

$$
\begin{aligned}
\rho_{0}^{c} \ddot{u}_{i}^{c}(\mathbf{X}, t)= & \sum_{I} \sum_{J} \frac{1}{\left|\Theta_{0}\right|} \frac{1}{2} \frac{\partial}{\partial X_{j}}\left(f_{i}^{I J} X_{j}^{I J}\right) \\
& +\left.\sum_{I} \sum_{J} \frac{1}{\left|\Theta_{0}\right|} \frac{1}{2} \frac{\partial f_{i}^{I J}}{\partial y_{j}^{I J}}\right|_{\mathbf{y}^{I J}=\mathbf{\varphi}^{I J}}\left(\frac{\partial u_{j}^{(1)}\left(\mathbf{X}, \mathbf{Y}^{J}, t\right)}{\partial X_{m}}+\frac{\partial u_{j}^{(1)}\left(\mathbf{X}, \mathbf{Y}^{I}, t\right)}{\partial X_{m}}\right) Y_{m}^{I J} .
\end{aligned}
$$

It can be shown that the second term of Equation (30) will vanish. Recalling Equation (3) yields the following:

$$
\mathbf{x}^{J I}=\mathbf{x}^{I}-\mathbf{x}^{J}=\mathbf{X}^{I}-\mathbf{X}^{J}+\mathbf{u}^{I}-\mathbf{u}^{J}=-\mathbf{x}^{I J}=\xi \boldsymbol{\varphi}^{J I}+\xi^{2} \boldsymbol{\psi}^{J I}(\mathbf{X}, \mathbf{Y}, t)+\ldots
$$

where

$$
\begin{aligned}
\varphi_{i}^{J I} & =F_{i j}^{c}(\mathbf{X}, t) Y_{j}^{J I}+u_{i}^{(1)}\left(\mathbf{X}, \mathbf{Y}^{I}, t\right)-u_{i}^{(1)}\left(\mathbf{X}, \mathbf{Y}^{J}, t\right)=-\varphi_{i}^{I J} \\
\psi_{i}^{J I} & =\frac{\partial u_{i}^{(1)}\left(\mathbf{X}, \mathbf{Y}^{I}, t\right)}{\partial X_{j}} Y_{j}^{J I}+\frac{1}{2} \frac{\partial^{2} u_{i}^{c}(\mathbf{X})}{\partial X_{j} \partial X_{k}} Y_{j}^{J I} Y_{k}^{J I}=-\psi_{i}^{I J} .
\end{aligned}
$$

According to Newton's third law, we have:

$$
\mathbf{f}^{I J}=-\mathbf{f}^{J I}
$$

From Equations (32) and (33), we have the relationship

$$
\frac{\partial f_{i}^{I J}}{\partial y_{j}^{I J}}=-\frac{\partial f_{i}^{J I}}{\partial y_{j}^{I J}}=-\frac{\partial f_{i}^{J I}}{\partial\left(-y_{j}^{J I}\right)}=\frac{\partial f_{i}^{J I}}{\partial y_{j}^{J I}} .
$$

The summation of the second term of Equation (30) gives the following:

$$
\begin{aligned}
& \left.\sum_{I} \sum_{J} \frac{1}{\left|\Theta_{0}\right|} \frac{1}{2} \frac{\partial f_{i}^{I J}}{\partial y_{j}^{I J}}\right|_{\mathbf{y}^{I J}=\mathbf{\varphi}^{I J}}\left(\frac{\partial u_{j}^{(1)}\left(\mathbf{X}, \mathbf{Y}^{J}, t\right)}{\partial X_{m}}+\frac{\partial u_{j}^{(1)}\left(\mathbf{X}, \mathbf{Y}^{I}, t\right)}{\partial X_{m}}\right) Y_{m}^{I J} \\
& =\left.\sum_{I} \sum_{J} \frac{1}{\left|\Theta_{0}\right|} \frac{1}{2} \frac{\partial f_{i}^{I J}}{\partial y_{j}^{I J}}\right|_{\mathbf{y}^{I J}=\boldsymbol{\varphi}^{I J}}\left(\frac{\partial u_{j}^{(1)}\left(\mathbf{X}, \mathbf{Y}^{J}, t\right)}{\partial X_{m}}+\frac{\partial u_{j}^{(1)}\left(\mathbf{X}, \mathbf{Y}^{I}, t\right)}{\partial X_{m}}\right)\left(Y_{m}^{J}-Y_{m}^{I}\right) .
\end{aligned}
$$


For any pair $(I, J)$, which participates the summation, we have

$$
\begin{aligned}
\frac{1}{2} & \left.\frac{\partial f_{i}^{I J}}{\partial y_{j}^{I J}}\right|_{\mathbf{y}^{I J}=\mathbf{\varphi}^{I J}}\left(\frac{\partial u_{j}^{(1)}\left(\mathbf{X}, \mathbf{Y}^{J}, t\right)}{\partial X_{m}}+\frac{\partial u_{j}^{(1)}\left(\mathbf{X}, \mathbf{Y}^{I}, t\right)}{\partial X_{m}}\right)\left(Y_{m}^{J}-Y_{m}^{I}\right) \\
& +\left.\frac{1}{2} \frac{\partial f_{i}^{J I}}{\partial y_{j}^{J I}}\right|_{\mathbf{y}^{J I}=\mathbf{\varphi}^{I I}}\left(\frac{\partial u_{j}^{(1)}\left(\mathbf{X}, \mathbf{Y}^{I}, t\right)}{\partial X_{m}}+\frac{\partial u_{j}^{(1)}\left(\mathbf{X}, \mathbf{Y}^{J}, t\right)}{\partial X_{m}}\right)\left(Y_{m}^{I}-Y_{m}^{J}\right) \\
= & \left.\frac{1}{2} \frac{\partial f_{i}^{I J}}{\partial y_{j}^{I J}}\right|_{\mathbf{y}^{I J}=\boldsymbol{\varphi}^{I J}}\left(\frac{\partial u_{j}^{(1)}\left(\mathbf{X}, \mathbf{Y}^{J}, t\right)}{\partial X_{m}}+\frac{\partial u_{j}^{(1)}\left(\mathbf{X}, \mathbf{Y}^{I}, t\right)}{\partial X_{m}}\right)\left(Y_{m}^{J}-Y_{m}^{I}\right) \\
& +\left.\frac{1}{2} \frac{\partial f_{i}^{I J}}{\partial y_{j}^{I J}}\right|_{\mathbf{y}^{I J}=\mathbf{\varphi}^{I J}}\left(\frac{\partial u_{j}^{(1)}\left(\mathbf{X}, \mathbf{Y}^{I}, t\right)}{\partial X_{m}}+\frac{\partial u_{j}^{(1)}\left(\mathbf{X}, \mathbf{Y}^{J}, t\right)}{\partial X_{m}}\right)\left(Y_{m}^{I}-Y_{m}^{J}\right) \\
= & 0 .
\end{aligned}
$$

Finally, the coarse-scale equation of motion is expressed as follows:

$$
\rho_{0}^{c} \ddot{u}_{i}^{c}(\mathbf{X}, t)=\sum_{I} \sum_{J} \frac{1}{\left|\Theta_{0}\right|} \frac{1}{2} \frac{\partial}{\partial X_{j}}\left(f_{i}^{I J} X_{j}^{I J}\right) .
$$

One can rewrite Equation (37) as

$$
\begin{aligned}
& \rho_{0}^{c} \ddot{u}_{i}^{c}(\mathbf{X}, t)-\frac{\partial P_{i j}(\mathbf{X}, t)}{\partial X_{j}}=0 \\
& P_{i j}(\mathbf{X}, t)=\frac{1}{2\left|\Theta_{0}\right|} \sum_{I} \sum_{J} f_{i}^{I J} X_{j}^{I J} .
\end{aligned}
$$

Alternatively, we have the following:

$$
P_{i j}(\mathbf{X}, t)=\frac{1}{\left|\Theta_{0}\right|} \sum_{I=1}^{n} \sum_{J>I} f_{i}^{I J} X_{j}^{I J}
$$

where $n$ denotes the total number of particles in the unit cell. Equation (39) can be also derived in the current configuration. Considering the relationship between the first Piola-Kirchhoff stress and Cauchy stress, we have the following:

$$
\begin{aligned}
& \mathbf{P}=J \boldsymbol{\sigma} \cdot \mathbf{F}^{-T} \\
& \boldsymbol{\sigma}=\mathbf{P} \cdot \mathbf{F}^{T} / J
\end{aligned}
$$

where $J$ is the determinant of deformation gradient. Inserting Equation (39) into Equation (40) yields the following:

$$
\begin{aligned}
\sigma_{i j} & =\frac{1}{J} P_{i k} F_{j k}=\frac{1}{J} \frac{1}{\left|\Theta_{0}\right|} \sum_{I=1}^{n} \sum_{J>I} f_{i}^{I J} X_{k}^{I J} F_{j k} \\
& =\frac{1}{|\Theta|} \sum_{I=1}^{n} \sum_{J>I} f_{i}^{I J}\left(F_{j k} X_{k}^{I J}\right)
\end{aligned}
$$

where $|\Theta|$ denotes the volume of the unit cell in the current configuration. From Equations (17) and (18), we have the following:

$$
\begin{aligned}
\mathbf{x}^{I J}=\xi\left(\mathbf{F}^{c} \cdot \mathbf{Y}^{I J}+\mathbf{u}^{(1)}\right. & \left.\left(\mathbf{X}, \mathbf{Y}^{J}, t\right)-\mathbf{u}^{(1)}\left(\mathbf{X}, \mathbf{Y}^{I}, t\right)\right)+O\left(\xi^{2}\right) \\
& =\mathbf{F}^{c} \cdot \mathbf{X}^{I J}+O(\xi) .
\end{aligned}
$$


Inserting Equation (42) into Equation (41) yields the following:

$$
\sigma_{i j}=\frac{1}{|\Theta|} \sum_{I=1}^{n} \sum_{J>I} f_{i}^{I J}\left(F_{j k} X_{k}^{I J}\right)=\frac{1}{|\Theta|} \sum_{I=1}^{n} \sum_{J>I} f_{i}^{I J} x_{j}^{I J}+O(\xi) .
$$

According to Equations (39) and (41), both the first Piola-Kirchhoff stress and Cauchy stress can be derived from the multiscale asymptotic analysis. The coarse-scale problem that governs macroscopic continuum behavior and the unit cell problem that replaces the macroscopic phenomenological constitutive laws can be expressed in the current configuration, which read,

a. Coarse-scale problem

$$
\begin{aligned}
& \rho^{c} \ddot{\mathbf{u}}^{c}(\mathbf{x}, t)-\nabla_{\mathbf{x}} \cdot \boldsymbol{\sigma}^{c}=\mathbf{0} \\
& \boldsymbol{\sigma}^{c}(\mathbf{x}, t)=\frac{1}{|\Theta|} \sum_{I=1}^{n} \sum_{J>I} \mathbf{f}^{I J} \mathbf{x}^{I J}
\end{aligned}
$$

b. Unit cell problem

$$
\sum_{J} \mathbf{f}_{i}^{I J}+\mathbf{F}_{e x t, i}\left(\mathbf{u}^{I}\right)=\mathbf{0}
$$

where $\ddot{\mathbf{u}}^{c}$ denotes the coarse-scale acceleration; $\rho^{c}=\frac{1}{|\Theta|} \sum_{I} m^{I}$ and $|\Theta|$ are the coarse-scale density, and the unit cell volume in the current configuration, respectively. $n$ is the number of particles in the unit cell; $\boldsymbol{\sigma}^{c}$ is the coarse-scale Cauchy stress, and $\mathbf{x}^{I J}$ is the vector connecting the centers of two particles. $(\nabla \cdot)$ denotes the divergence operator, and superscript $c$ denotes the coarse-scale features. The Cauchy stress obtained in Equation (44)b is identical to the classical homogenized Cauchy stress obtained using the principle of virtual work [18, 37-39].

Note that the inertia term in Equation (4) only enters the coarse-scale equation of motion, whereas the unit cell problem remains quasi-static. This is because the coarse-scale wave length is assumed to be much larger than the RVE size. This approach is commonly used for low rates of loading and for short observation times [30, 44]. However, in those problems with high rates of loading and long observation times, particle interfaces in a granular media may cause reflection and refraction of stress waves, giving rise to dispersion and attenuation of waves within material microstructure [45], which cannot be accounted for by the approach developed in this paper.

\subsection{Coarse-scale problem: FEM}

In the macroscopic continuum scale, the trajectories of individual particles are not considered. Instead, we associate each coarse-scale material point with a representative elementary volume or unit cell in which effective continuum properties can be derived. As a result, the coarse scale displacement field is interpolated by the finite element basis function and possesses $C^{0}$ continuity. The initial and boundary conditions for the coarse-scale problem described by Equation (44) are given as:

$$
\begin{gathered}
\mathbf{u}^{c}(\mathbf{x}, 0)=\mathbf{0} ; \quad \dot{\mathbf{u}}^{c}(\mathbf{x}, 0)=\mathbf{0} \\
\mathbf{u}^{c}(\mathbf{x}, t)=\overline{\mathbf{u}}(\mathbf{x}, t) \quad \text { on } \quad \partial \Omega^{u} ; \quad \boldsymbol{\sigma}^{c} \cdot \mathbf{n}=\overline{\mathbf{t}} \quad \text { on } \quad \partial \Omega^{\mathrm{t}}
\end{gathered}
$$

where the essential (displacement) boundary $\partial \Omega^{u}$ and the natural (traction) boundary $\partial \Omega^{t}$ satisfy $\partial \Omega^{u} \cup \partial \Omega^{t}=\partial \Omega$ and $\partial \Omega^{u} \cap \partial \Omega^{t}=0$, as shown in Figure 2 ; $\dot{\mathbf{u}}^{c}$ is the velocity vector; $\overline{\mathbf{u}}$ and $\overline{\mathbf{t}}$ represent prescribed displacements and tractions on $\partial \Omega^{u}$ and $\partial \Omega^{t}$, respectively. And $\mathbf{n}$ is the unit outward norm of the boundary.

By applying the weight-residual method, the weak form corresponding to the coarse-scale problem in Equation (44)a can be written as follows. 
Find $\mathbf{u}^{c}(\mathbf{x}, t) \in \mathscr{U}$ in $\Omega$ such that

$$
\int_{\Omega}\left(\nabla_{\mathbf{x}} \mathbf{w}^{c}\right): \sigma^{c} d \Omega=\int_{\partial \Omega^{t}} \mathbf{w}^{c} \cdot \overline{\boldsymbol{t}} d \Omega-\int_{\Omega} \rho^{c} \mathbf{w}^{c} \cdot \ddot{\boldsymbol{u}}^{c} d \Omega \quad \forall \mathbf{w}^{c} \in \mathscr{W}
$$

where the trial and test function spaces are defined as follows:

$$
\begin{aligned}
\mathscr{U} & =\left\{\mathbf{u}^{c} \mid \mathbf{u}^{c} \in \mathscr{H}^{1}, \mathbf{u}^{c}=\overline{\boldsymbol{u}}^{c} \text { on } \partial \Omega^{u}\right\} \\
\mathscr{W} & =\left\{\mathbf{w}^{c} \mid \mathbf{w}^{c} \in \mathscr{H}^{1}, \mathbf{w}^{c}=0 \text { on } \partial \Omega^{u}\right\}
\end{aligned}
$$

where $\mathbf{u}^{c}$ and $\mathbf{w}^{c}$ are the coarse-scale trial and test functions, respectively, and $\mathscr{U}^{1}$ is the Sobolev space of order one. The Galerkin form is obtained by specifying the finite dimensional space for both the trial function and interpolated coarse displacement field. Here, we consider the case in which both the trial function and the displacement field are spanned by the same basis function. As a result, the coarse-scale trial and test functions

$$
\begin{aligned}
{ }^{h} \mathbf{u}^{c}(\mathbf{x}, t) & =\sum_{A \in \mathbb{N}} \mathbf{N}_{\mathrm{A}}(\mathbf{x}) \mathbf{d}_{\mathrm{A}}^{c}(t) \\
{ }^{h} \mathbf{w}^{c}(\mathbf{x}, t) & =\sum_{A \in \mathbb{N}} \mathbf{N}_{\mathrm{A}}(\mathbf{x}) \mathbf{w}_{\mathrm{A}}^{c}(t)
\end{aligned}
$$

which yields a semidiscrete momentum equation as follows:

$$
\mathbf{M} \ddot{\mathbf{d}}^{c}(t)=\mathbf{f}^{\mathrm{ext}}(t)-\mathbf{f}^{\mathrm{int}}\left(\mathbf{d}^{c}(t)\right)
$$

where $\mathbf{N}_{\mathrm{A}}$ is the basis function corresponding to node $\mathrm{A} ; \mathbb{N}$ represents the set of nodes in the mesh and $\mathbf{d}_{\mathrm{A}}^{c}(t)$ and $\mathbf{w}_{\mathrm{A}}^{c}(t)$ denote the nodal degrees-of-freedom of trial and test functions, respectively. The superscript $h$ represents the discretized quantities; $\mathbf{d}^{c}$ and $\ddot{\mathbf{d}}^{c}$ are the coarse-scale nodal displacements and accelerations, respectively, and $\mathbf{M}, \mathbf{f}^{\text {int }}$, and $\mathbf{f}^{\text {ext }}$ are the coarse-scale mass matrix, internal force, and external force vectors, respectively. In the numerical implementation, we employ the Voigt's notation such that second-order tensors are stored as column vectors. As a result, the internal and external force vector and the mass matrix can be written as a function of the shape function $\mathbf{N}$, and the discrete symmetric gradient operator $\mathbf{B}$, that is as follows,

$$
\mathbf{M}=\int_{\Omega} \rho^{c} \mathbf{N}^{T} \mathbf{N} d \Omega ; \quad \mathbf{f}^{\mathrm{int}}=\int_{\Omega} \mathbf{B}^{T} \boldsymbol{\sigma}^{c} d \Omega ; \quad \mathbf{f}^{\mathrm{ext}}=\int_{\Omega} \mathbf{N}^{T} \bar{t}^{c} d \Omega
$$

where $\boldsymbol{\sigma}^{c}$ is the coarse-scale Cauchy stress defined by Equation (44b) but stored in the Voigt form. In the present work, the continuum coarse-scale problem in Equation (51) is integrated using explicit central difference method [46]. Lumped mass is used in the coarse-scale simulations. Unlike the conventional macroscopic finite element approach, the constitutive responses are not obtained from macroscopic constitutive law but rather from homogenized responses of particle assemblies associated with every quadrature point in the finite element mesh.

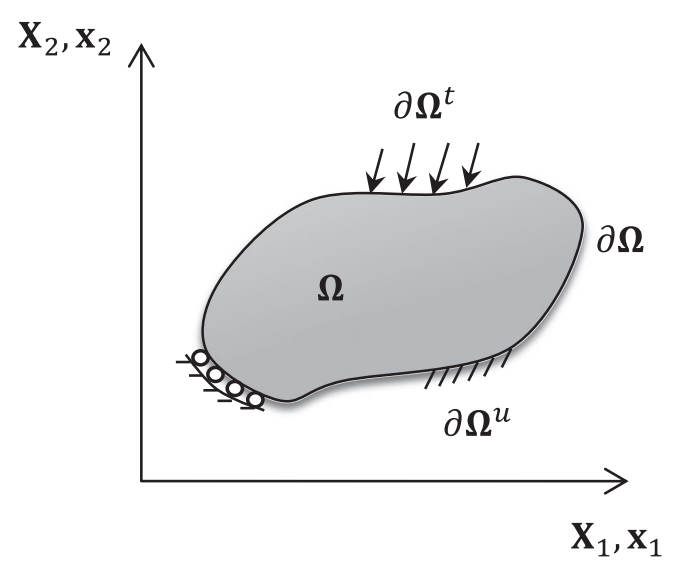

Figure 2. Schematics of the coarse-scale boundary value problem (in the initial configuration). 


\subsection{Discrete element unit cell problem}

Consider a collection of particles within the interior domain of a cuboidal unit cell. This collection of particles is surrounded by an exterior layer of one-particle thickness. This exterior layer deforms periodically in space and may interact with the particles inside the unit cell, but its motion is completely controlled by the prescribed periodic boundary conditions provided by the coarse-scale problem. The particle-to-particle and particle-to-boundary interactions are both simulated via discrete element method, and the quasi-static macroscopic responses of the unit cell are subsequently homogenized from each unit cell and passed to the coarse-scale solver.

The quasi-static solution of the unit cell problem in Equation (45) can be obtained by seeking the steady-state solution of an explicit dynamic relaxation DEM problem with incremental loading steps or by directly solving the nonlinear system of equations via an implicit scheme. In practice, the implicit scheme is rarely used for DEM problems. As the nonlinearity of responses may stem from both the nonlinear and path dependent contact laws and the changes of topologies of grain contacts, it is easier to implement and use explicit scheme to obtain the quasi-static solution of DEM assemblies. The dynamic relaxation problem can be viewed as an iterative process in which pseudo-dynamics processes are emulated in an artificial time scale. This iterative process is considered complete when a deformed configuration of the granular assembly with all the forces and moment in equilibrium is found. Using numerical examples to provide evidences, Bardet and Proubet [47] show that both mass scaling and viscous damping can be used to enhance computational efficiency (by reducing number of iterative steps) without significantly altering the approximated quasi-static configurations of the DEM assemblies. In this paper, an explicit central difference leap-frog pseudo-time integrator and artificial damping are both employed to obtain the quasi-static solution [15].

2.3.1. Contact models for discrete element simulations in unit cells. A simplified contact model, which employs Hertz-Mindlin contact law and Coulomb's friction law with viscous damping, is used to represent the particle contact mechanism. Cohesive bonding and rolling resistance between the particles are not considered in this paper. Incremental changes to the normal and tangential contact forces, $f_{n}^{f}$ and $f_{t}^{f}$, at each contact are determined by the particle shear modulus $G_{g}^{f}$, Poisson's ratio $v^{f}$, radii of the contacting grains $R_{1}$ and $R_{2}$, and the fine-scale normal and tangential displacements at the contact, $d \delta^{f}$ and $d s^{f}[48]$ as follows,

$$
\begin{array}{cc}
d f_{n}^{f}=k_{n}^{f} d \delta^{f} ; & k_{n}^{f}=\frac{\sqrt{2} G_{g}^{f} \sqrt{R^{e}}}{1-v^{f}}\left(\delta^{f}\right)^{1 / 2} \\
d f_{t}^{f}=k_{t}^{f} d s^{f} ; & k_{t}^{f}=\frac{2 \sqrt{2} G_{g}^{f} \sqrt{R^{e}}}{2-v^{f}}\left(\delta^{f}\right)^{1 / 2}
\end{array}
$$

where $\delta^{f}$ is the indentation at the contact and $R^{e}$ is the effective radius,

$$
R^{e}=\frac{2 R_{1} R_{2}}{R_{1}+R_{2}}
$$

Superscript $f$ denotes fine-scale features. The fine-scale tangential force is governed by the friction coefficient $\mu^{f}$, such that $\left|f_{t}^{f}\right| \leqslant \mu^{f} f_{n}^{f}$. For stabilization, a viscous damping force is employed

$$
f_{s, v i s}^{f}=C^{f} \dot{s}^{f}
$$

where $C^{f}$ and $\dot{s}^{f}$ are the viscosity and the tangential sliding velocity at contact. In case of slow, quasi-static loading conditions, the mass damping $c^{I}$ and contact damping $C^{f}$ must be sufficient to dissipate high frequency vibrational modes without impeding particle motion that arise from particle interactions or the boundary conditions. 
2.3.2. Dynamic relaxation scheme of unit cell problem. For a given unit cell, the stress of the DEM assemblies depends on the fine-scale material parameters, microstructural attributes, such as particle size distribution, spatial heterogeneity, and the loading path and history that lead to the current configuration $[49,50]$. If the wavelength of the traveling signal is significantly larger than the dimensions of the particles, then the quasi-static stress homogenized from the forces and branch vectors of the quasi-static configuration of unit cell can be used to update stress measures of the macroscopic dynamics problem without introducing significant errors.

Various criteria have been proposed to detect the quasi-static state of DEM dynamic relaxation problem $[49,50,51]$. In this work, we use the unbalanced force index $I_{u f}$ introduced by $\mathrm{Ng}$ [52],

$$
I_{u f}=\sqrt{\frac{\frac{1}{n_{p}} \sum_{1}^{n_{p}}(\text { unbalanced forces })^{2}}{\frac{1}{n_{c}} \sum_{1}^{n_{c}}(\text { contact forces })^{2}}}
$$

where $n_{p}$ and $n_{c}$ denote the number of particles and number of contacts, respectively. The size of the pseudo-time or load step is calibrated such that the unbalanced force index $I_{u f}$ is always kept smaller than a threshold value, for example, 0.01 . This treatment ensures that the artificial damping introduced for dynamics relaxation does not significantly affect the simulated friction angle and shear strength. Mass scaling is also used to enable the usage of larger pseudo-time step and to reduce computational cost. In each pseudo-time step, the incremental displacement is prescribed to the particles at the boundary of the unit cell such that the unit cell deforms with the strain increment prescribed by the macroscopic problem. One particular interesting finding from Andrade and Tu [28] is that the granular assemblies with more particles typically require a small strain increment and more loading steps to maintain static equilibrium. Notice that if an identical quasi-static boundary value problem is simulated by both DEM and DEM-FEM models, the pure DEM model will require much more increment loading steps than the DEM-FEM counterpart [28]. In this sense, the DEM-FEM model can be considered as a divide-and-conquer tactic tool to coarsen load increments and increase efficiency of numerical simulations for large scale quasi-static granular mechanics problems.

2.3.3. Numerical algorithms for the nonlocal multiscale discrete-continuum model. The two-scale problem, consisting of the discrete unit cell problem subjected to periodic boundary conditions and the coarse-scale equations of motion, is two-way coupled. In this section, we focus on algorithmic details. The two-scale problem described by Equations (44) and (45) is solved sequentially as follows:

(i) Solve the dynamics relaxation problem at coarse scale using a co-rotational finite element model and compute the coarse-scale strain increment of each local quadrature.

(ii) Obtain the nonlocal coarse-scale strain increment using the staggered nonlocal operator and evaluate the nonlocal corotational strain increment.

(iii) Prescribing periodic boundary conditions to the unit cells on the nonlocal corotational coarsescale strain increment of the corresponding nonlocal quadrature.

(iv) Obtain new static equilibrium states of the granular assemblies compatible to the prescribed boundary condition via DEM and compute the corotational coarse-scale Cauchy stress by Equation (44b).

(v) Transform the corotational coarse-scale Cauchy stress to the fixed global frame and compute residual vector. Go back to (i) for next step.

To account for the geometric nonlinear effect, we employ a corotational formulation [53] where a local corotational coordinate frame, denoted by, is attached to each finite element quadrature point and rotated with the deforming material. To preserve the characteristic length scale and eliminate pathological mesh dependence, we use a staggered nonlocal operator that explicitly introduces an intrinsic length scale and thus limits the shear band thickness when strain localization occurs. The interaction radius, denoted by $\mathrm{R}$, is assumed to be an intrinsic material property. 
In step (i), the coarse-scale incremental strain $\Delta \varepsilon_{n+1 / 2}^{\mathrm{c}}(\mathbf{x}, t)$ is obtained from the solution of the coarse-scale problem at each quadrature point, in each time step. Because of strong size dependence that has been observed in granular materials, we define a nonlocal coarse-scale strain increment $[35,36]\left\langle\Delta \varepsilon_{n+1 / 2}^{\mathrm{c}}\left(\mathbf{x}_{I}\right)\right\rangle_{R}$ as follows:

$$
\begin{aligned}
\left\langle\Delta \varepsilon_{n+1 / 2}^{c}\left(\mathbf{x}_{I}\right)\right\rangle_{R} & =\sum_{\xi_{J} \in Q_{I}} \alpha^{*}\left(\mathbf{x}_{I}, \xi_{J}\right) \phi^{*}\left(\xi_{J}\right) \\
\phi^{*}\left(\xi_{J}\right) & =\left\{\begin{array}{l}
\Delta \varepsilon_{n+1 / 2}^{c}\left(\xi_{I}\right) \text { if } \xi_{J}=\mathbf{x}_{I} \\
\Delta \varepsilon_{n+\alpha}^{c}\left(\xi_{J}\right) \text { if } \xi_{J} \neq \mathbf{x}_{I}
\end{array}\right.
\end{aligned}
$$

where $\Delta \boldsymbol{\varepsilon}_{n+\alpha}^{c}$ denotes the coarse-scale strain computed on the fly, that is, $\alpha$ is $\pm(1 / 2)$, which represents either previous or current time step. $\Delta \varepsilon_{n+1 / 2}^{c}$ is the coarse-scale strain at the current time $t_{n+1}$; $\mathbf{x}_{I}$ denotes the position of the current quadrature point $I$, and $Q_{I}$ is a set of quadrature points $\xi_{J} \in Q_{I}$ adjacent to point $\mathbf{x}_{I}$ that satisfies the following:

$$
\left\{Q_{I}|| \mathbf{x}_{I}-\xi_{J} \mid \leqslant R, \forall \xi_{J} \in Q_{I}\right\}
$$

where $R$ denotes the characteristic radius. $\alpha^{*}\left(\mathbf{x}_{I}, \xi_{J}\right)$ is defined in Appendix B.

Prior to exerting $\left\langle\Delta \varepsilon_{n+1 / 2}^{c}\left(\mathbf{x}_{I}\right)\right\rangle_{R}$ onto the unit cell, it is rotated to the corotational frame, to yield the nonlocal corotational coarse-scale strain increment $\left\langle\Delta \boldsymbol{\varepsilon}_{n+1 / 2}^{\Re}\left(\mathbf{x}_{I}\right)\right\rangle_{R}$ as follows:

$$
\left\langle\Delta \varepsilon_{n+1 / 2}^{\Re}\left(\mathbf{x}_{I}\right)\right\rangle_{R}=\left(\Re_{n+1 / 2}^{c}\left(\mathbf{x}_{I}\right)\right)^{T} \cdot\left\langle\Delta \varepsilon_{n+1 / 2}^{c}\left(\mathbf{x}_{I}\right)\right\rangle_{R} \cdot\left(\Re_{n+1 / 2}^{c}\left(\mathbf{x}_{I}\right)\right)
$$

where $\mathfrak{R}_{n+1 / 2}^{c}\left(\mathbf{x}_{I}\right)$ denotes the coarse-scale rotation obtained from the polar decomposition of the coarse-scale deformation gradient $\mathbf{F}_{n+1 / 2}^{c}\left(\mathbf{x}_{I}\right)$ at a quadrature point $\mathbf{x}_{I}$ defined as follows:

$$
\mathbf{F}_{n+1 / 2}^{c}\left(\mathbf{x}_{I}\right)=\frac{\partial \mathbf{x}_{n+1 / 2}}{\partial \mathbf{X}},
$$

and $\mathbf{x}_{n+1 / 2}$ is the coordinate at the midstep. Instead of directly prescribing the local strain increment as periodic boundary conditions applied on the boundaries of unit cells, the proposed model employs the coarse-scale strain increment $\left\langle\Delta \varepsilon_{n+1 / 2}^{\Re}\left(\mathbf{x}_{I}\right)\right\rangle_{R}$ obtained from the macroscopic solver to obtain the corotational coarse-scale Cauchy stress $\sigma_{n+1}^{\Re}(\mathbf{x}, t)$ through Equation (44)b using DEM, which is then rotated back to the global Cartesian coordinate system as follows.

$$
\sigma_{n+1}^{c}=\left(\Re_{n+1}^{c}\right) \cdot \sigma_{n+1}^{\Re} \cdot\left(\Re_{n+1}^{c}\right)^{T} .
$$

The resulting coarse scale stress $\sigma_{n+1}^{c}(\mathbf{x}, t)$ is passed back to coarse-scale finite element engine to continue the iterative process. The aforementioned nonlocal discrete-continuum model is implemented by integrating FOOF [54], a macroscale FEM solver, and OVAL [55], a microscale DEM solver. The numerical algorithm that links between the macro-scale and micro-scale solvers is shown in the flow chart in Figure 3. At each nonlocal quadrature point in the FEM mesh, the FEM solver executes the DEM unit cell problem in the corotational frame subjected to the nonlocal coarsescale incremental strain $\left\langle\Delta \varepsilon_{n+1 / 2}^{\Re}\left(\mathbf{x}_{I}\right)\right\rangle_{R}$. The fine-scale DEM solver evolves the discrete unit cell using explicit time integration to compute the coarse-scale Cauchy stress required to advance the coarse-scale problem. The particle arrangement, that is, particle positions, velocities, and contact information, at the end of the coarse-scale time step are stored, to allow the new DEM simulation to begin from the final state of the previous time step. Each integration point has its own "restart" out-of-core file. The numerical implementation of the corotational formulations is illustrated in Appendix B. 


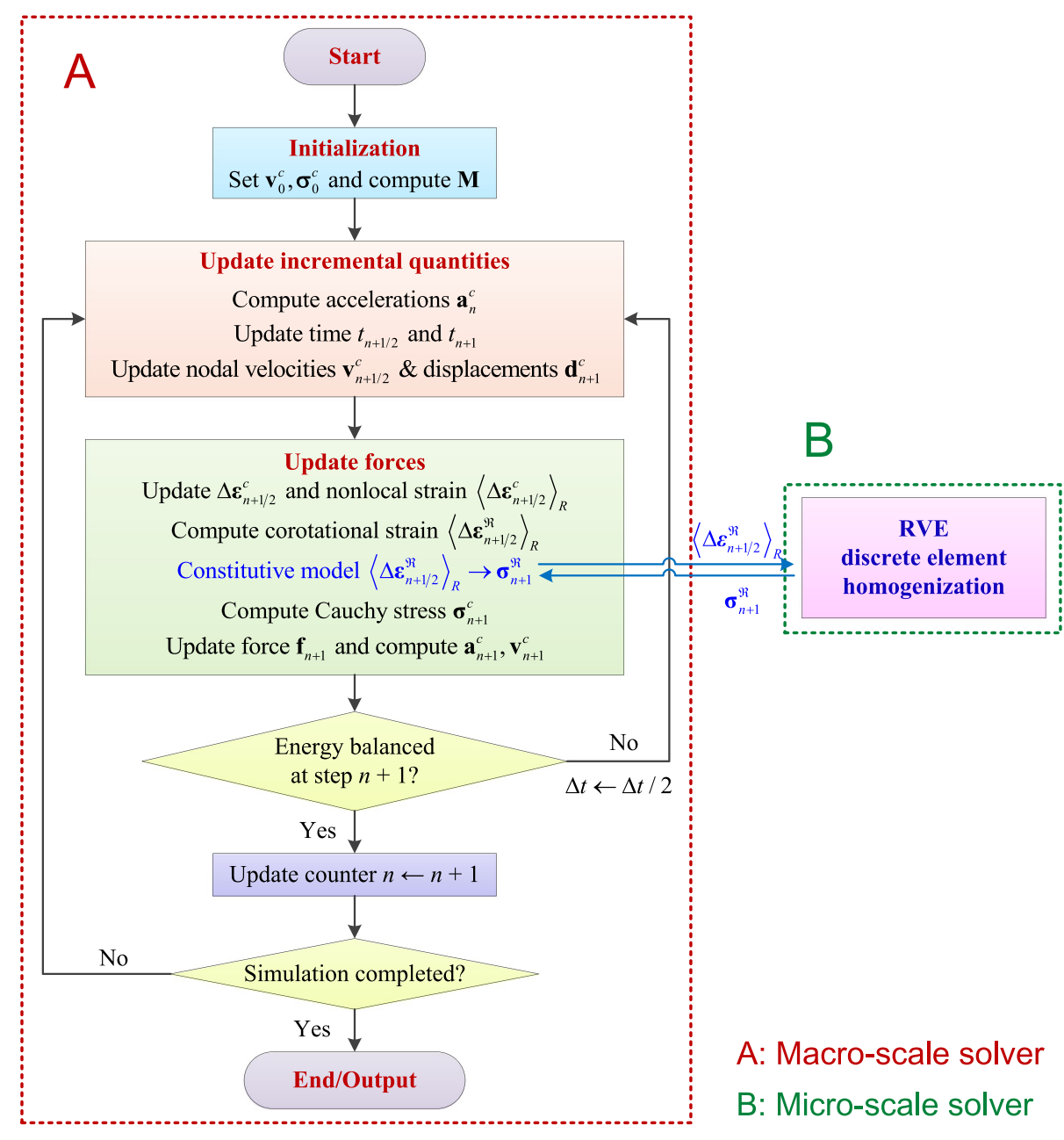

Figure 3. The framework integrating FEM and DEM solvers for the two-scale problem.

2.3.4. Temporal Stability of the coupled explicit DEM-FEM problems. The objective of the unit cell dynamics relaxation problems is to obtain stress measures from the granular configurations in the static equilibrium. As a result, both the mass and damping coefficients used in the DEM are not related to the actual physical quantities but are parameters manipulated by the users to obtain nonoscillatory overall responses. While the original physical meanings of the mass and damping are lost in dynamics relaxation problem, both the explicit dynamics and dynamics relaxation problem still share the same form of governing equations and can be integrated in time by the conditionally stable explicit scheme. The critical pseudo-time step of the DEM problem therefore takes the same form as a damped mass-spring system, that is as follows,

$$
\Delta t_{n}^{f} \leqslant \Delta t_{c r}^{f}=\frac{2}{\omega_{\max }^{f}}\left(\sqrt{1+\xi^{f^{2}}-\xi^{f}}\right)
$$

where $\Delta t_{c r}^{f}$ denotes the critical time step of the macroscopic problem; $\xi^{f}$ denotes the fraction of critical damping corresponding to the highest natural frequency of the granular system $\omega_{\max }^{f}$. Assuming that there is no rotational stiffness introduced in the DEM contact model, the natural frequencies of the translational and rotational vibration of each particle read as follows (Oñate and Rojek [56]),

$$
\omega_{n}^{f}=\sqrt{\frac{k_{n}^{f}}{m^{f}}} ; \omega_{\theta}=\sqrt{\frac{k_{\theta}^{f}}{I_{\text {sphere }}}}=\sqrt{\frac{5 k_{t}^{f}}{2 m^{f}}} ; k_{\theta}^{f}=k_{t}^{f} R^{2} ; I_{\text {sphere }}=\frac{2}{5} m^{f} R^{2}
$$


where $I_{\text {sphere }}$ is the rotational inertia of the spherical particles and $m^{f}$ is the mass of the particle. The critical time step of the DEM simulation can be obtained via Equation (63) where the highest natural frequency is approximated by $\omega_{\max }^{f} \approx \max \left(\omega_{n}^{f}, \omega_{\theta}\right)$. In practice, the DEM dynamics relaxation problems are often conducted with a fixed time step, while mass of the particles is tuned to ensure the stability between two pseudo-time steps.

On the other hand, the stable time step for the coarse-scale system is governed by the highest frequency $\left(\omega_{\max }^{c}\right)$ of the course-scale incremental finite element problem as follows:

$$
\Delta t_{n}^{c} \leqslant \Delta t_{c r}^{c}=\frac{2}{\omega_{\max }^{c}}\left(\sqrt{1+\left(\xi^{c}\right)^{2}}-\xi^{c}\right)
$$

where $\Delta t_{c r}^{c}$ denotes the critical time step of the macroscopic problem; $\xi^{c}$ denotes the fraction of critical damping corresponding to the highest natural frequency of the macro-scale FEM model. The critical step defined in Equation (65) also applies to the case where mass scaling and damping are applied to the macroscopic problem to obtain static equilibrium solution. Nevertheless, the critical macroscopic time step of the dynamic relaxation DEM-FEM problem is larger than that of the explicit DEM-FEM dynamics problem, as the damping and mass scaling are likely to filter out high frequency responses. Note that the critical time step of the fine-scale DEM and coarse-scale FEM can be related by the following,

$$
\Delta t_{c r}^{c}=\alpha \Delta t_{c r}^{f} ; \alpha=\psi \frac{\omega_{\max }^{f}}{\omega_{\max }^{c}} ; \psi=\frac{\sqrt{1+\left(\xi^{c}\right)^{2}}-\xi^{c}}{\sqrt{1+\left(\xi^{f}\right)^{2}}-\xi^{f}}
$$

where $\alpha$ is the optimal time step ratio between the fine-scale and coarse-scale systems. Notice that because the highest value frequency of the fine system is typically the higher one, $\alpha$ is usually larger than one.

\section{NUMERICAL EXAMPLES}

In this section, four numerical examples are presented to demonstrate the accuracy, efficiency, and versatility of the proposed multiscale method in predicting the mechanical behavior of granular materials. Examples shown in this section provide evidences that the multiscale DEM-FEM model is able to replicate the single-scale DEM benchmark results. Refinement study indicates that the thickness of shear bands predicted by the nonlocal multiscale model is not sensitive to the mesh sizes of the continuum model.

\subsection{Unit cell}

The initial configuration of the unit cell is given in Figure 4. The unit cell was compacted into a dense and isotropic assembly from an initially sparse random arrangement of particles, which contains 4096 spherical grains with diameters ranging from 0.43 to $1.18 \mathrm{~mm}$. The porosity after compaction was 0.338 , with an initial average coordination number of 5.6 contacts per particle. The initial normal stresses in all directions were nearly equal to the mean stress of $416 \mathrm{kPa}$. The initial response of the unit cell is assumed to be macroscopically homogeneous.

Periodic boundaries were employed on all sides of the unit cell in the numerical examples presented in this paper. Such boundaries impose kinematic constrains on each boundary particle, allowing grains to pass from the parallelepiped unit cell domain to a fictitious adjacent one and simulate an infinitely periodic (repeated) system. Period boundaries are computationally advantageous over the rigid platens for providing a more uniform particle fabric throughout the assembly [24, 57]. Previous work done by [58-60] has found that homogenized responses inferred from periodic cell are less sensitive to boundary effects.

The micro-model parameters given in Table I were used as input microscopic parameter in the following numerical examples, where shear modulus and Poisson's ratio are the parameters for Hertz-Mindlin contact model. The mechanical properties of the material were obtained from pure 
DEM tests on the unit cell, for example, the uniform compression test was used to get bulk modulus and the simple shear test to get the shear modulus [61]. The measured mechanical properties are shown in Table II.

Accuracy and efficiency of the proposed multiscale technique rely crucially on the appropriate selection of the size of the unit cell so that it remains a RVE. In particular, the unit cell size must be small enough to ensure computational efficiency but large enough to remain representative. One common approach to estimate the size of the RVE is to vary the sizes of the unit cells and study the scale of fluctuation of the coarse-scale properties [30, 62-65]. Meier et al. [66] and Guo and Zhao [27] applied this approach to discrete element models. Guo and Zhao [27] generated multiple granular assembles composed of different numbers of particles and studied the least amount of particles required to maintain the isotropy of the fabric tensor. They concluded that at least 400 particles required to constitute an RVE composed of particles of poorly graded grain size distribution. In the numerical examples presented in this paper, the grain assembles used to calculate the Gauss point responses are composed of at least 4000 particles and have been examined in previous study to ensure the isotropy of the initial fabric tensor.

All simulations presented in this section are conducted with a three-dimensional eight-node hexahedral finite element integrated via the one-point Gaussian quadrature rule. The hour-glass control stabilization procedure in [67-69] is used to eliminate spurious zero-energy modes in the macroscopic finite element model. While the usage of one-point integration rule is a rather simple and well-known numerical treatment, the benefits for the DEM-FEM coupling scheme are significant. It improves both the speed and the accuracy of the multiscale simulations by cutting $87.5 \%$ of the expensive 3D DEM unit cell simulations in $[26,27]$ that might cause volumetric locking problems otherwise.

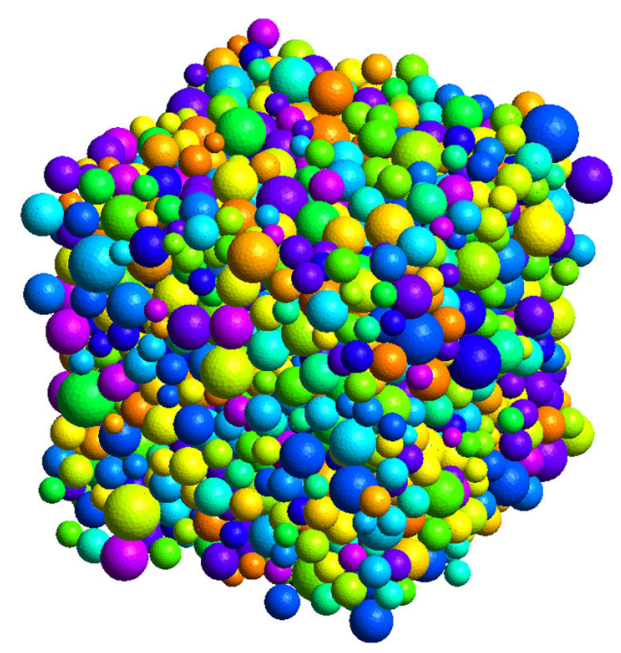

Figure 4. The initial configuration of the unit cell.

Table I. Model parameters for discrete element method computation.

\begin{tabular}{ll}
\hline 29.0 & \multicolumn{1}{c}{ Shear modulus $G_{g}^{f}(\mathrm{GPa})$} \\
\hline 0.15 & Poisson ratio $v^{f}$ \\
0.50 & Coefficient of friction at particle contacts \\
0.00 & Viscosity coefficient for translational body damping \\
0.00 & Viscosity coefficient for rotational body damping \\
$0.12(0.00$ for Section 3.5$)$ & Viscosity coefficient for contact damping \\
\hline
\end{tabular}


Table II. Material properties estimated from unit cell tests [54, 55].

\begin{tabular}{lccc}
\hline Young's modulus & Poisson's ratio & Bulk modulus & Shear modulus \\
$E(\mathrm{MPa})$ & $v$ & $K(\mathrm{MPa})$ & $G(\mathrm{MPa})$ \\
\hline 584 & 0.2 & 335 & 241 \\
\hline
\end{tabular}
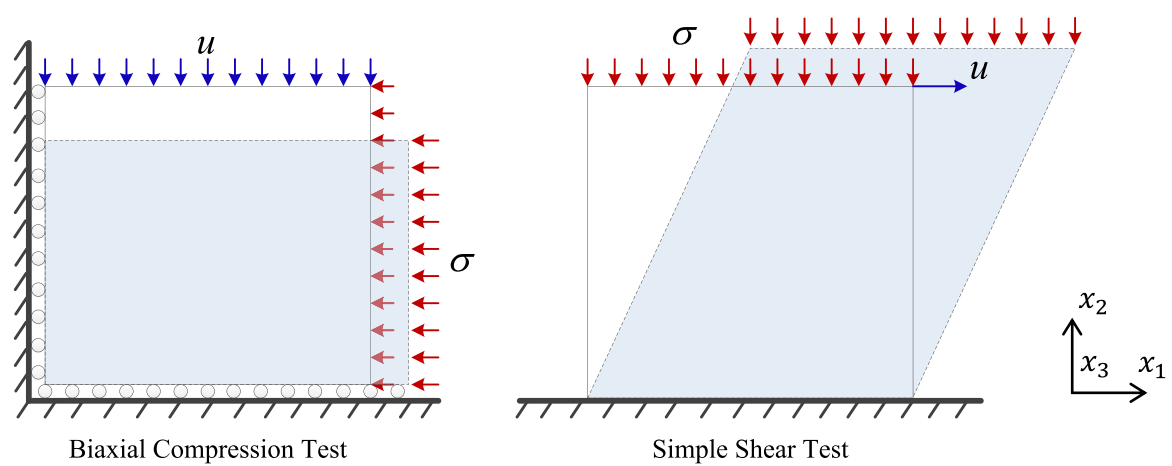

Figure 5. The boundary conditions for single element biaxial compression (left) and simple shear (right) tests.

\subsection{One element verification tests}

The multiscale model was first verified by comparing the global response of a single element (biaxial compression test and simple shear test) against the RVE response from pure DEM tests. This verification procedure is first introduced in [27] to test the accuracy and robustness of the developed information-passing DEM-FEM coupling scheme at the material point level.

The single element and its boundary conditions are depicted in Figure 5. In the simple shear test, the single element was subjected to a shear loading by keeping the confining pressure on the top to be constant. In the biaxial compression test, a vertical displacement was applied on the top surface, and constant confining pressure was exerted on the right surface. In both cases, the constant confining pressures were $416 \mathrm{kPa}$, which was identical with the initial mean stress of the unit cell.

The comparison of stress-strain curves is shown in Figure 6 for the one-element tests using the multiscale DEM-FEM model and single-scale DEM. It can be observed that the multiscale approach gives almost identical prediction with those from the DEM simulations. This example verifies that the proposed multiscale discrete-continuum approach can replicate the elasto-plastic response of granular material accurately.

\subsection{One-dimensional wave propagation in a dry granular column}

The modeling of wave propagation in granular materials, such as wave attenuation and dispersion, is an active research area with implications for seismic soil-structure interaction and foundation vibration [70-74]. Continuum-based analytical and computational modeling techniques have been widely used to investigate the seismic wave propagation $[75,76]$. The path dependent responses of soil are often captured via macroscopic phenomenological models $[7,73,74,77]$. While the cost of the continuum simulations is relatively low, the physical underspin of the phenomenological approach in the softening regimes is weak. On the other hand, dynamics discrete element simulations are also utilized by a number of researchers to study the micromechanics of wave propagation in granular matters [78-81]. The upshot of the discrete element approach is the availability of microstructural information. However, the high computational cost of DEM often limits the size of the simulations and thus making DEM not feasible to simulate field-scale site responses.

This numerical example is designed to demonstrate the potential of using the newly established DEM-FEM method to overcome the shortcomings of both continuum and discrete element approaches. To the best knowledge of the authors, this is the first time that information-passing 

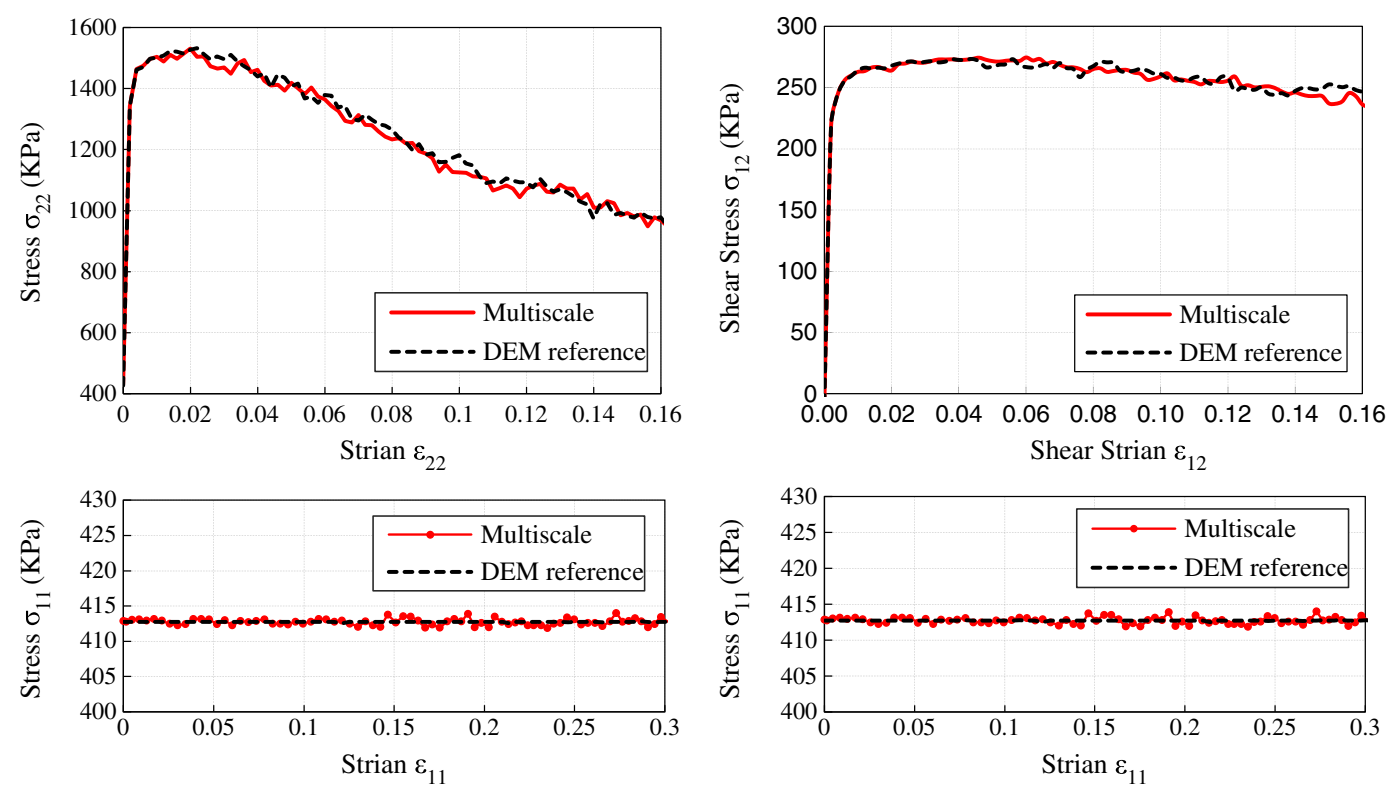

(a) Biaxial Compression

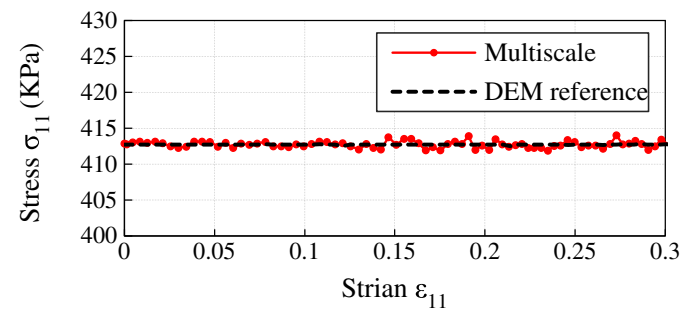

(b) Simple Shear

Figure 6. The comparison of stress-strain curves between the multiscale simulation and DEM reference in the one element tests: (a) biaxial compression and (b) simple shear.

DEM-FEM scheme was being used to simulate dynamics responses of granular layers. The boundary value problem used in this example is commonly used for predicting site responses in geotechnical earthquake engineering and often referred as the (one-dimensional) soil column problem $[71,73,74]$. It consists of a stratified granular layer rests on a bedrock that is assumed to be rigid at $x_{1}=0$. Assume that the in-place dimensions $\left(x_{2}\right.$ and $\left.x_{3}\right)$ are infinite relative to the granular layer thickness, body wave emanating from the dynamic excitation of the bedrock will propagate along the $x_{1}$ axis in a one-dimensional fashion [73, 74]. As a result, we impose kinematic constraints $\varepsilon_{22}=\varepsilon_{33}=\varepsilon_{23}=0$ to enforce the wave propagating only along the $x_{1}$ direction. In the numerical simulation, we discretize a spatial domain of $100 \mathrm{~mm} \times 10 \mathrm{~mm} \times 10 \mathrm{~mm}$ with equal-sized eight-node trilinear brick element, as shown in Figure 7. The left hand side boundary of the model was totally fixed, and two pairs of periodic boundaries were applied to the two opposite faces of the one-dimensional soil column orthogonal to the $x_{2}$ and $x_{3}$ directions, while the kinematic constraints $\varepsilon_{22}=\varepsilon_{33}=\varepsilon_{23}=0$ are imposed by enforcing zero in-plane relative displacement on plane original to the $x_{1}$ axis (cf. [73, 74]). At the macroscopic scale, the geometric domain was discretized by 10 finite elements of equal sizes. The density of the model was $\rho=1650 \mathrm{~kg} / \mathrm{m}^{3}$. The right hand side of the model was subjected to a vertically displacement-controlled sinusoidal cyclic load with frequency of $f=10 \mathrm{kHz}$ and maximum shearing displacement amplitude of $a=0.1 \mathrm{~mm}$. The time duration of the whole simulation was $1 \mathrm{~ms}$, and the coarse-scale time increment was taken as $\Delta t=1 \mu \mathrm{s}$.

The homogenized shear and compressive stress-strain responses of the quadrature point of Element 9 are shown in Figure 8(a). We observe that the hysteresis and the dissipative responses of non-cohesive frictional granular materials are captured. Unlike previous macroscopic approaches where microstructural attributes are only taken into account indirectly via the evolution of internal variables, the multiscale approach is able to provide important microstructural measurements, such as coordination number and porosity of each unit cell as shown in Figure 8(b). This numerical experiment suggests that the proposed multiscale model is capable of modeling the dynamic responses of granular materials subjected to cyclic loadings within limited computational resources. This is a major departure from the previous DEM modeling efforts, in which particles are enlarged artificially for the sake of reducing the computational cost [82]. 


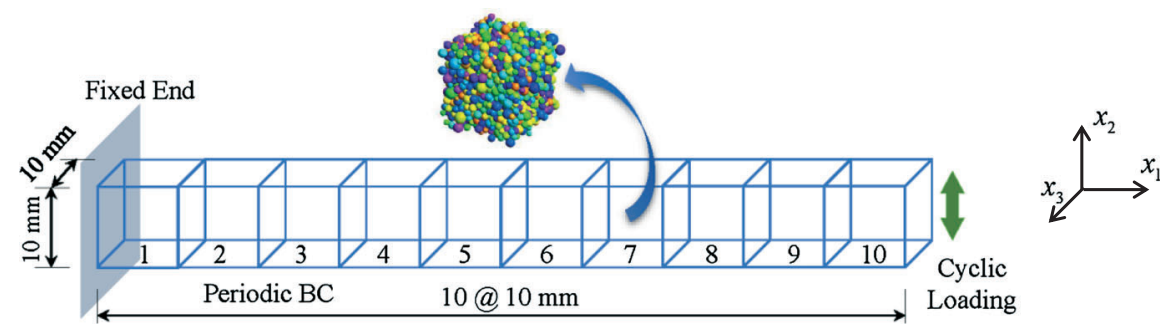

Figure 7. The boundary condition and macroscopic mesh of the sample for wave propagation in a dry granular column.
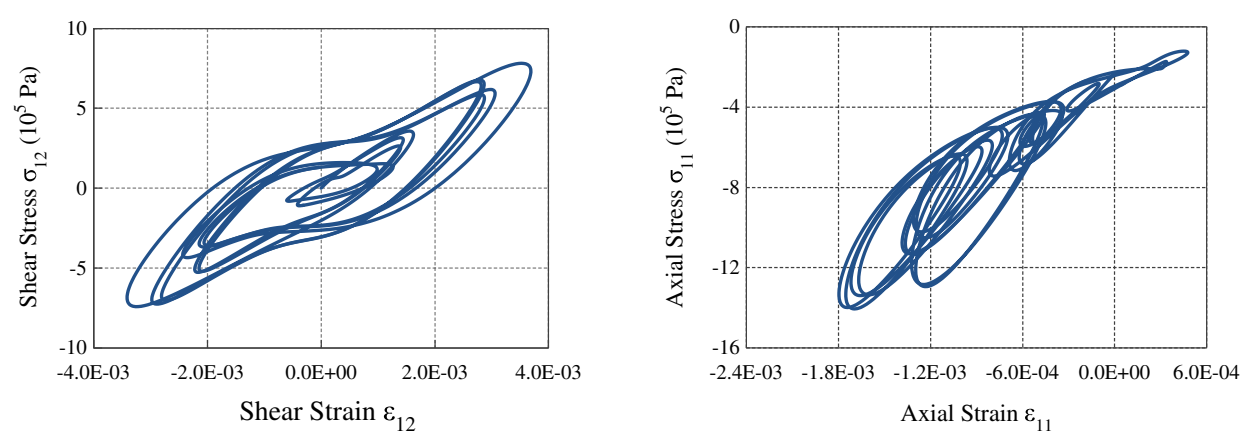

(a) Stress-strain curves
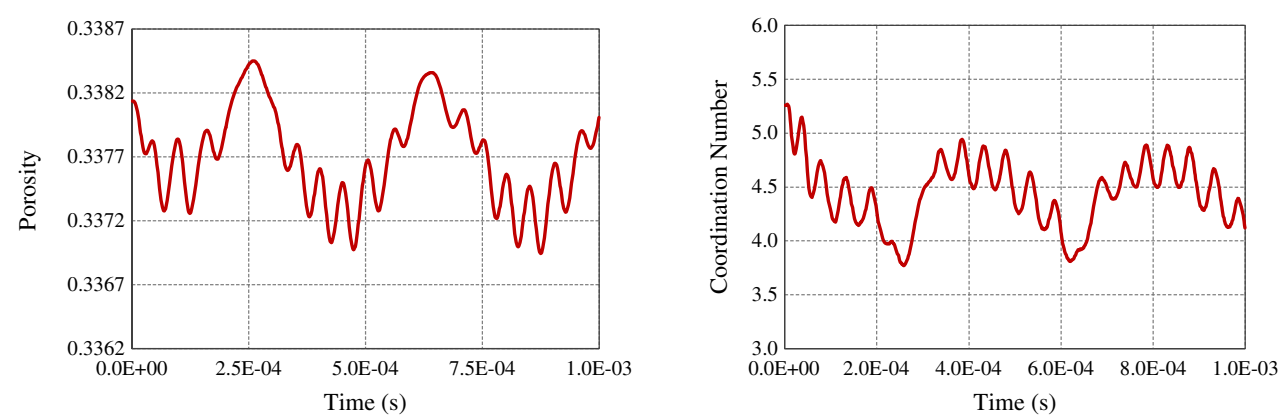

(b) Porosity and coordinate number of grain contacts versus time

Figure 8. Dynamic responses of RVE associated with the Gauss point at Element 9:(a) Stress-strain curves and (b) Porosity and coordinate number of grain contacts versus time.

\subsection{Shear band in a simple shear simulation on a dense grain assembly}

In this example, the proposed model was further validated by a simple shear test on a dense grain assembly. Multiscale simulation results were compared with those from a single-scale DEM simulation conducted in [48]. The geometries and loading conditions are schematically shown in Figure 9. The model dimensions were $50.6 \mathrm{~mm} \times 118 \mathrm{~mm} \times 12.7 \mathrm{~mm}$. It was fixed at its bottom and sheared horizontally at its top boundary. Periodic boundary conditions were applied to the four surrounding surfaces in $x_{1}$ and $x_{3}$ directions to ensure a shear band develops along the full $x_{1}-x_{1}$ width when passing across the periodic side boundaries. The domain of the numerical specimen was discretized into a uniform coarse mesh with $12 \times 5 \times 1$ elements. The grain assemblies assigned to all quadrature points are initially identical, and thus, the numerical specimen is macroscopically homogeneous. As a result, the shear band in a simple shear test may occur at any height, which makes the location of the band unpredictable. The previous DEM simulation results reported in [48] indicate that a shear band forms along a horizontal plane, which is located at about three-quarters of the assembly height from its fixed boundary, a result likely to be caused by subtle imperfection within the grain 
assembly. To match the location of shear band in the DEM and DEM-FEM simulations, we artificially imposed an imperfection inside the shear band to break the symmetry and homogeneity of the sample. The unit cell with imperfection was created by randomly taking out 25 particles from the original unit cell. It should be noted that the initial state of the unit cell, for example, mean stress, porosity, and coordination number, needs to be identical with those of the grain assembly in the single-scale DEM test. To maintain a quasi-static loading condition, the mass-scaling technique was used in the coarse-scale finite element method for the entire model throughout the simulation.

Figures 10 to 13 show the macroscopic mechanical behavior of the shear band during shear loading with constant vertical stress $p_{0}=416 \mathrm{kPa}$. In the multiscale simulation results, the overall shear strain was computed from the horizontal displacement $u_{1}$ measured at the top surface divided by the initial height $h=118 \mathrm{~mm}$ while the shear stress was obtained from the reaction force at the fixed end in the direction of shearing divided by the area of the bottom surface. Good agreements are observed in these figures between the results predicted by both the multiscale model and the DEM. For example, Figure 10 shows the shear stress-strain response of the grain assembly. Both shear stress and mean stress (see Figures 10 and 11) reach their peak value and remaining nearly level until $\varepsilon_{12}=6 \%$, followed by an abrupt softening between the strains $\varepsilon_{12}=6 \%$ and $\varepsilon_{12}=8 \%$. Then at $\varepsilon_{12}=8 \%$ to $20 \%$, shear stress and mean stress fluctuate at the critical state where porosity remains constant. Plastic dilatancy is observed in Figure 12 where the porosity of the assembly increases monotonically even as the compressive mean stress increases. As shear loading proceeds, porosity converges to a constant level after the shear stress reaches to its residual condition. This increase of volume is ascribed to the re-arrangement of particles in dense spherical packings and dense sands as they rise up over neighboring particles [48, 83]. Figure 13 shows the average coordination number of the entire assembly. By comparing the multiscale and pure DEM simulations, we observe that the

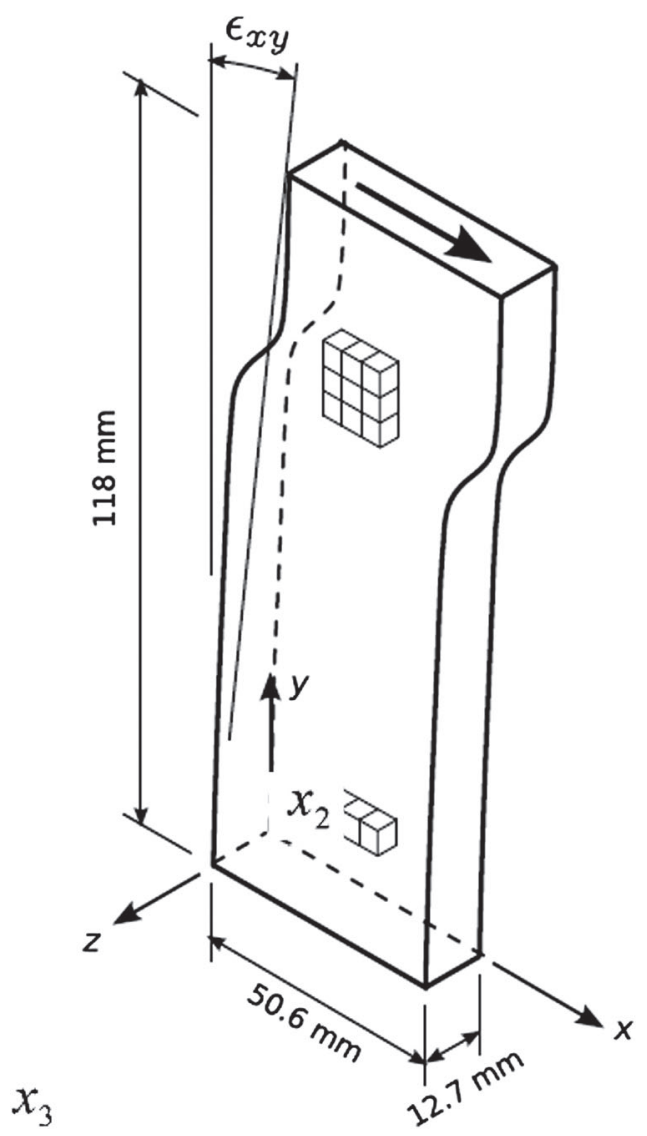

Figure 9. Geometry of the domain and the boundary $\operatorname{cor}_{x_{1}}$ ions for the simple shear test [77]. 


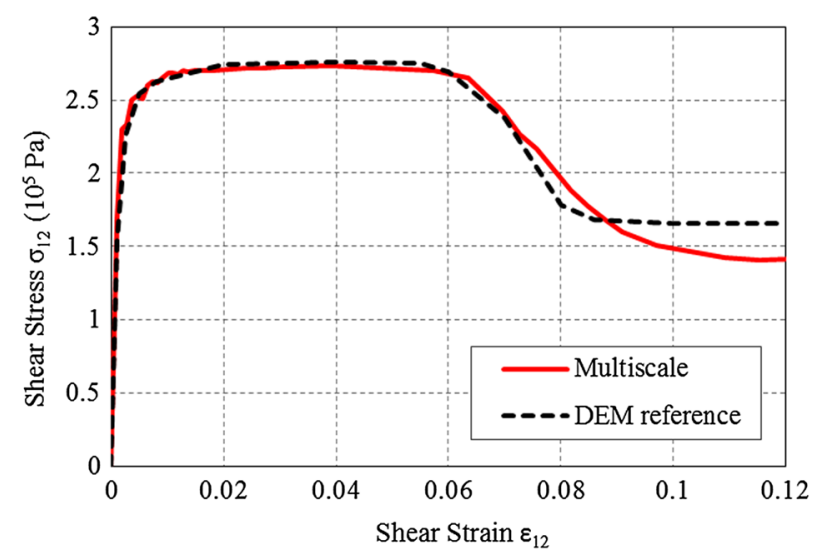

Figure 10. Shear stress-strain response during simple shear loading.

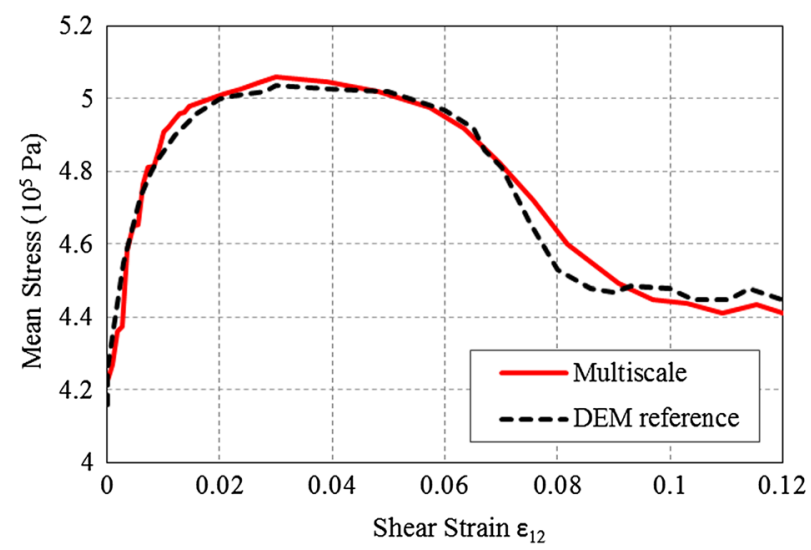

Figure 11. Mean stress versus shear strain during simple shear loading.

multiscale model is able to replicate the evolution of coordination number, which decreases rapidly in early, prepeak stress stage, and eases down to a nearly constant in a gently sloping stretch throughout the subsequent peak, softening, and residual periods.

The deformed configurations and averaged porosity along the assembly height at shear strain $\varepsilon_{12}=12 \%$ obtained from multi-scale and single-scale simulations are compared in Figure 14(a). The porosity curve obtained from the multiscale simulation is composed of 12 discrete points, each of which is an average of the porosities of a row of macroscopic element. Both the multiscale and DEM simulations indicate that the shear band thickness is about $13-16 \mathrm{~mm}$, a quantity of great importance, and will be used as a measure of the characteristic length in the size effect studies in the next example. The $\mathrm{L}_{2}$ norms of the Euler angles of each particle in the unit cells inside and outside the shear band are plotted in Figure 14(b) to analyze the evolutions of microstructural attributes inside the deformation band. Consistent to the finding in the single-scale benchmark, the multiscale model predicts that particles inside the shear band rotate more than those outside the band. No grain-scale deformation band was found inside the unit cells of the multiscale model.

Given the same computational resource, the CPU time for the multiscale simulation for the simple shear test with the current coarse mesh is 22 hours, which is much shorter than the CPU time used to complete the pure DEM counterpart (about one month). Therefore, it is evident that the proposed multiscale approach can reduce the computational cost significantly while making accurate prediction of the mechanical behaviors for granular materials. This enormous saving in simulation time is attributed to (1) the efficient bridging of the different spatial scales, (2) the introduction of multiple time step scheme, which allows the coarse-scale problem to evolve at a much larger time step without 


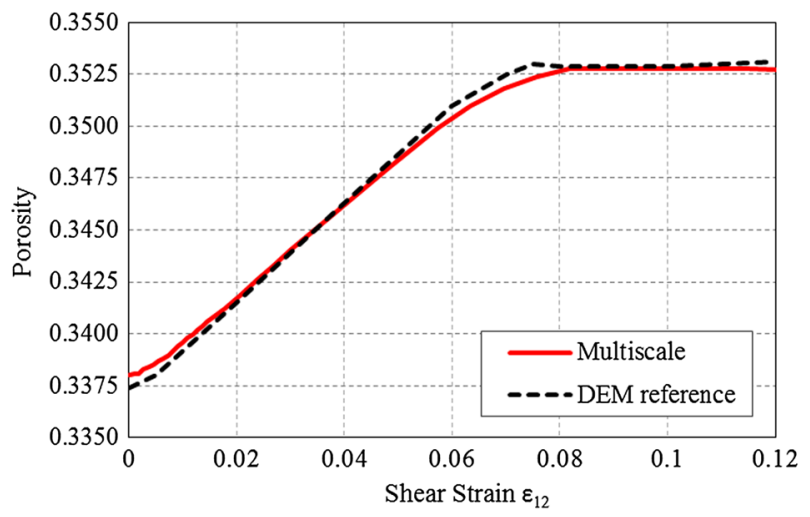

Figure 12. Porosity versus shear strain during simple shear loading.

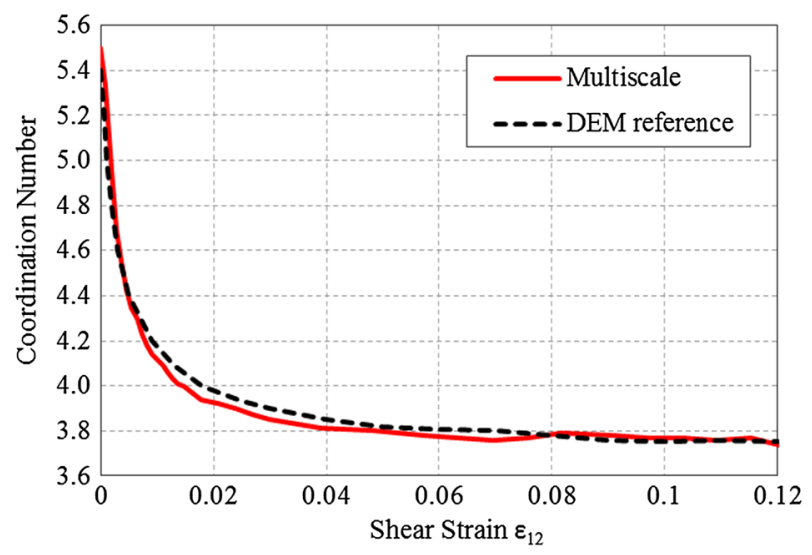

Figure 13. Coordination number versus shear strain during simple shear loading.

causing stability issues, and (3) the usage of the reduced integration elements with hourglass control, which both reduces solution times substantially and eliminates shear locking while maintaining spatial stability

\subsection{Shear band in a plane strain biaxial compression test}

Strain localization is of great importance to engineering applications, as it is often a precursor to progressive failure in granular materials. If no length scale is introduced, the onset of strain localization in numerical simulations may cause the loss of ellipticity (for static cases) or hyperbolicity (for dynamic cases) of the boundary value problem. This undesired ill-posedness may lead to pathological mesh dependence. To circumvent this issue in continuum models, various localization limiters have been introduced. They include (i) nonlocal or gradient models of which the constitutive response is governed by a gradient or integral of at least one internal variable(s) or strain measure [84-86], (ii) a ratedependent constitutive law [46], and (iii) formulations that permit displacement discontinuities [87-91].

Pathological mesh size dependency has also been observed in the previous multiscale DEMFEM-coupling simulations [26, 27]. This pathological mesh size dependency is due to the illposedness of the macroscopic finite element model [46].

The proposed multiscale approach remedies this situation via a modified staggered nonlocal approach. To test whether this staggered nonlocal operator successfully introduces an intrinsic length scale and limits the shear band thickness when strain localization occurs, two sets of biaxial compression tests were carried out using the local and nonlocal multiscale model, respectively. The geometry, boundary conditions, and the macroscopic meshes of the model are shown in Figure 15(a). 


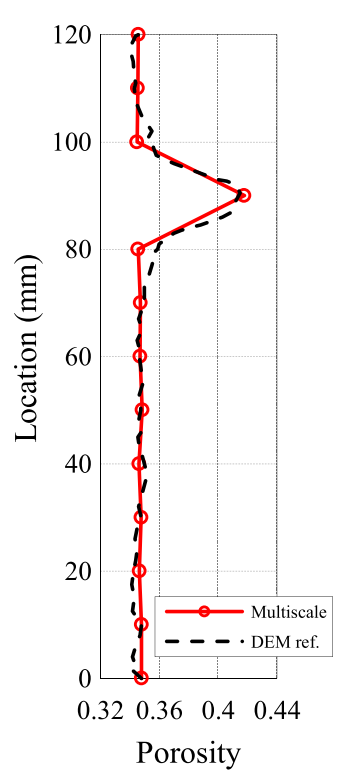

(a) Porosity versus height

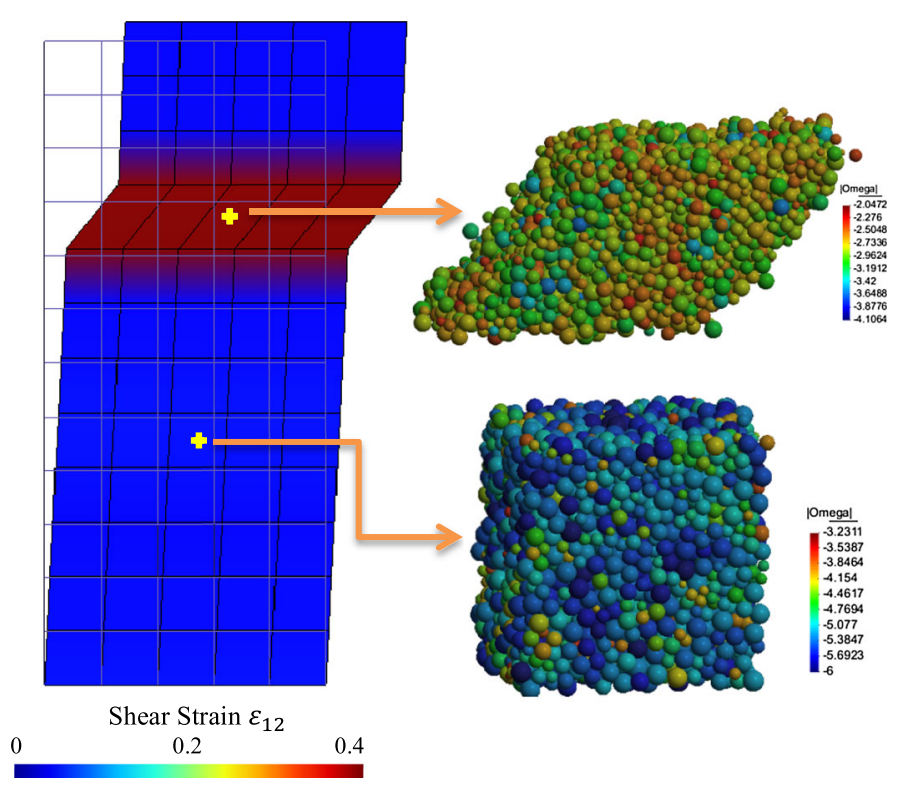

(b) Deformed configuration of RVEs inside and outside the shear band

Figure 14. Profiles of (a) porosity and (b) deformed configurations of the two RVEs inside and outside the shear band at strain $\varepsilon_{12}=12 \%$, with color in each particle indicated the magnitude the rotation measured by the norms of the rotation tensors.

The dimensions of the specimen were $40 \mathrm{~mm} \times 80 \mathrm{~mm} \times 5 \mathrm{~mm}$. A displacement-controlled vertical load was monotonically applied on the top surface of the model. The confining pressure applied on horizontal boundaries remained constantly throughout the loading. The asymmetric boundary condition was used at the bottom surface in order to break symmetry and to initiate the localization. Three finite element meshes depicted in Figure 15(b) were considered. For the nonlocal multiscale model, the characteristic length was chosen to be $2 R=13.5 \mathrm{~mm}$, which is the same with the unit cell size and is also consistent with the shear band width observed in the previous example.

In order to examine whether the nonlocal staggered scheme regularizes softening responses, we remove the potential regularizations attributed from the rate-dependence of the contact laws. In other words, damping parameters for grain contacts are set to be zero in both the local and nonlocal simulations, as shown in Table I. The global stress-strain responses predicted by local and nonlocal multiscale models from three different meshes are shown in Figures 16 and 18, respectively. In both figures, all three meshes yield almost identical pre-peak stress-strain behaviors and similar peak stresses. The slight kink shown in the elastic regime is due to the wave reflection from the bottom boundary of the model, which may be removed by reducing the tolerance of the dynamics relaxation scheme in Equation (57). As loading proceeded, strain localization emerged and finally developed into a shear band. The constitutive responses obtained from the local multiscale model exhibit obvious mesh dependence in the post-bifurcation region, as shown in Figure 16. On the other hand, the post-bifurcation stress-strain responses obtained from the nonlocal multiscale model, as shown in Figure 18, are nearly mesh independent. The softening responses of the three meshes in the nonlocal tests are almost identical, and the three curves converge even after shear bands are fully developed. Figures 17 and 19 compare the contour plots of vertical compressive strain $\varepsilon_{22}$ for three meshes using local and nonlocal multiscale models, respectively. Figure 17 shows that the local multiscale model leads to different band thickness when varying mesh size and that the finer meshes lead to narrower shear bands and higher intensity strains when using the local multiscale model. Meanwhile, the nonlocal multiscale model produces shear bands of similar widths, provided that enough degrees of freedom are used to interpolate the tip of the shear band as shown in the last two cases in Figure 19. 


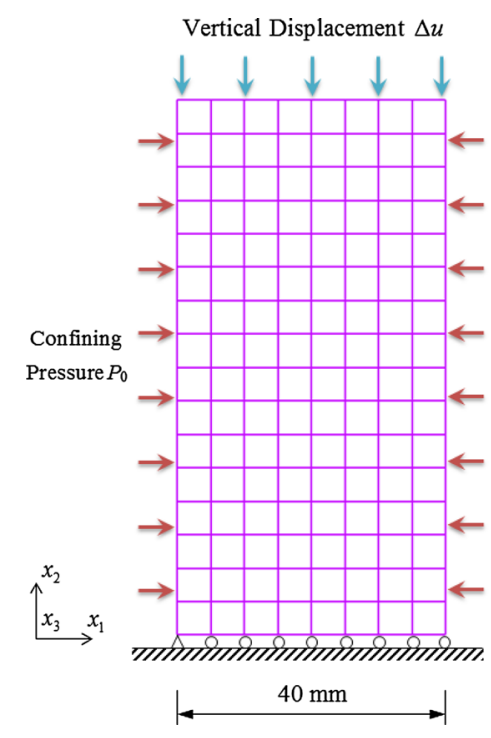

(a) Model geometry and boundary conditions

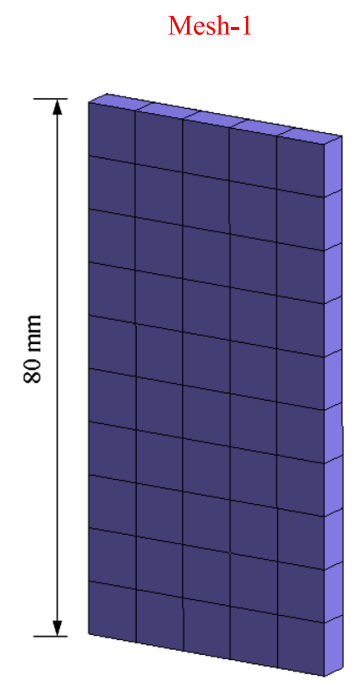

Mesh-2
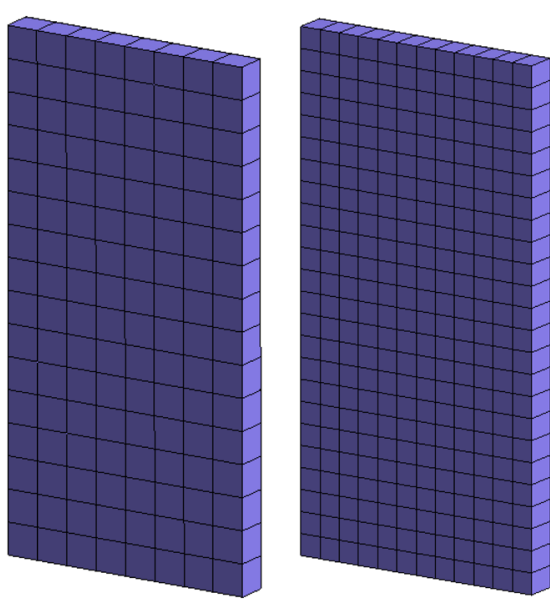

(b) Three meshes with $10 \times 5 \times 1,16 \times 8 \times 1$ and $24 \times 12 \times 1$ elements, respectively

Figure 15. (a) The spatial domain and boundary conditions; (b) three meshes considered in the biaxial compression tests.

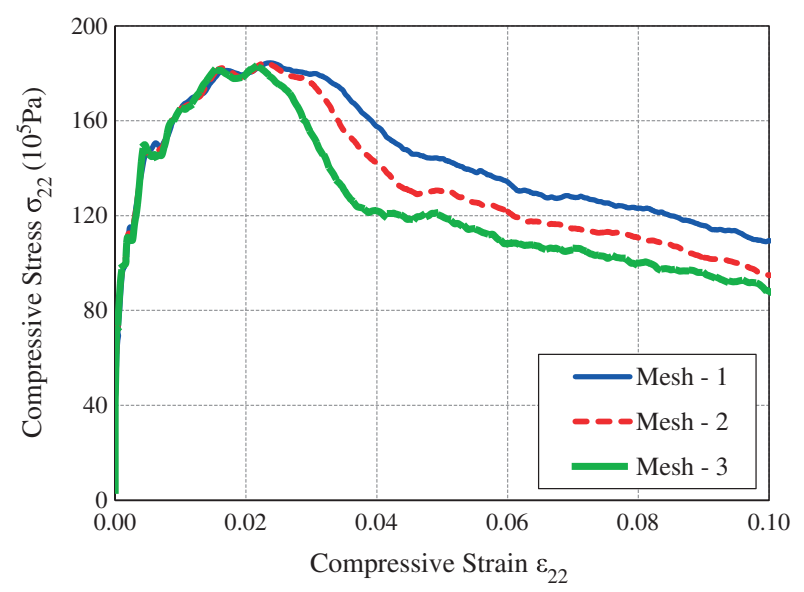

Figure 16. Compressive stress versus compressive strain curves for different meshes using local multiscale model.

By comparing the stress-strain curves and the compressive strain distribution shown in Figures 18-19, one may observe that the nonlocal multiscale scheme is able to deliver more consistent responses in the refinement study. Notice that the post-peak branch of the finest mesh of the stress-strain curve not always giving the softest response. This is attributed to the fact that there are no sufficiently material points in the coarse mesh to obtain an accurate integration for the nonlocal strain. As a result, the length scale of the coarse mesh is slightly larger than the fine mesh counterparts. In all numerical simulations, we found no shear band generated in the DEM assemblies. This absence of grain-scale shear band can be attributed by the particle shapes, the absence of rotational stiffness, lack of enrichment mode for the macroscopic finite element, and the usage of periodic boundary condition as opposed to minimal kinematic boundary conditions. Detail examinations of these factors are out of the scope of the current study but will be considered in the future. 


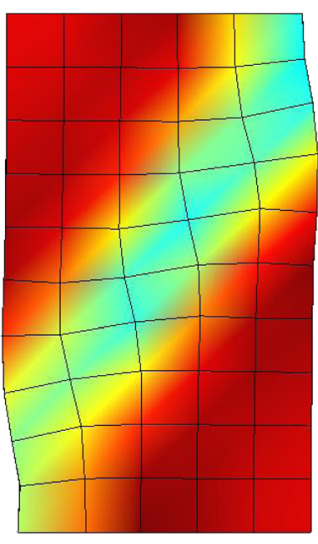

Compressive Strain $\varepsilon_{22}$

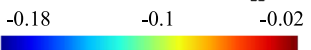

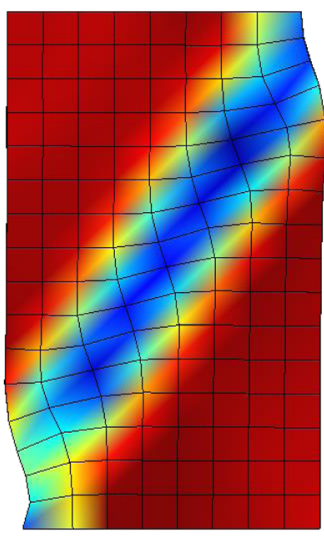

Compressive Strain $\varepsilon_{22}$

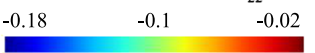

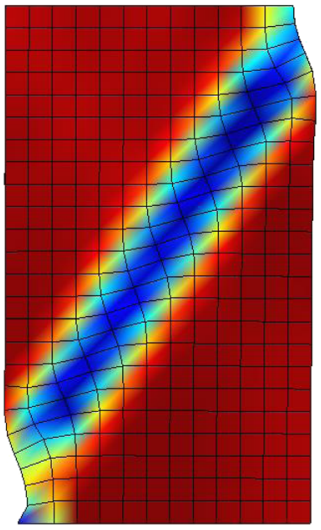

Compressive Strain $\varepsilon_{22}$ $\begin{array}{lll}-0.18 & -0.1 & -0.02\end{array}$

Figure 17. Contour plots of compressive strain for three meshes at $\varepsilon_{22}=6 \%$ (local multiscale model)

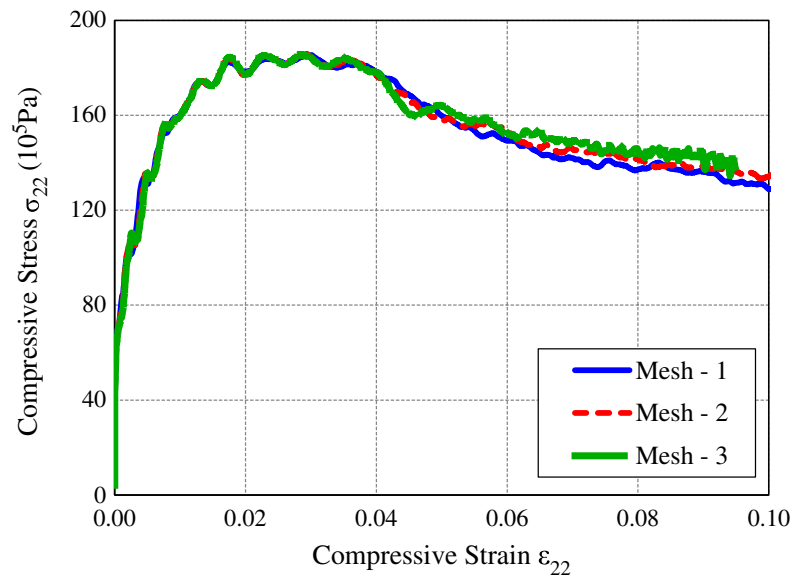

Figure 18. Compressive stress versus compressive strain curves for different meshes using nonlocal multiscale model.

\section{CONCLUSIONS}

In this paper, we present a nonlocal multiscale discrete-continuum model for granular materials. The proposed multiscale model effectively bridges two spatial scales, the coarse (continuum) scale and the fine (discrete) scale, by an information-passing coupling scheme based on the GMH theory. Each nonlocal quadrature point in the coarse-scale mesh is associated with a unit cell consisting of a granular assembly. The nonlocal strain obtained from the FEM solvers is converted into periodic boundary conditions for the grain-scale simulations occurred in the unit cells, which in return provide the nonlocal constitutive update at the macroscopic level via upscaling. This proposed model is verified via four benchmark problems. Good agreement has been observed by comparing the numerical solutions obtained via the multiscale DEM-FEM model with the single-scale DEM benchmark. The numerical examples demonstrate that the proposed multiscale discrete-continuum model is capable of reproducing both the dynamic and quasi-static behaviors of granular materials, and simulation results obtained for bifurcation problems are practically mesh size independent. The multiple spatial scales and multi-step framework also present a significant cost reduction compared with the direct DEM simulations. 

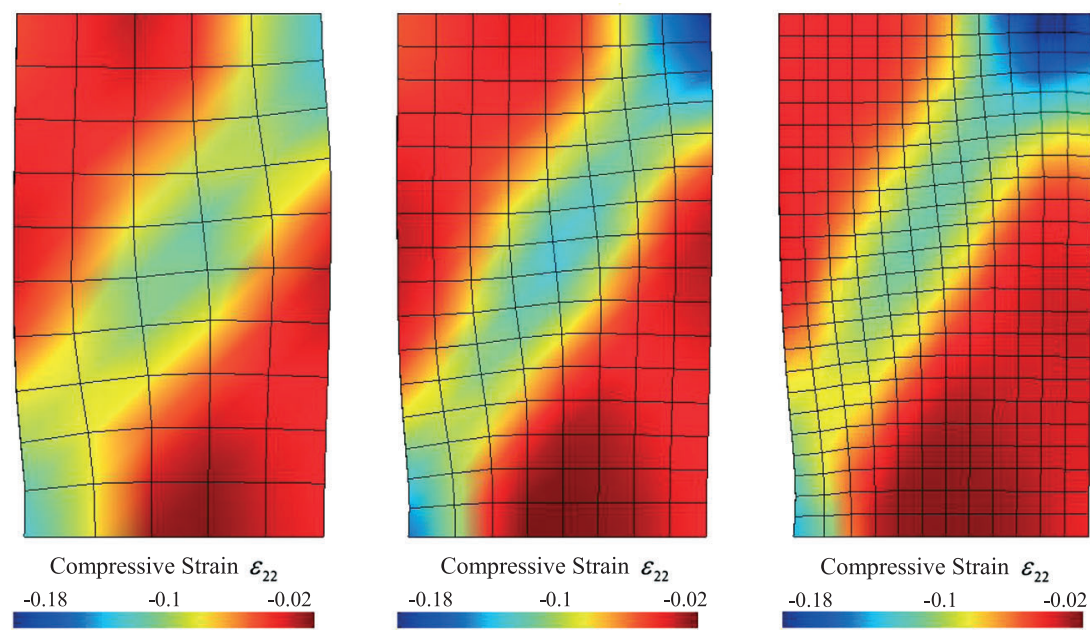

Figure 19. Contour plots of compressive strain for three meshes at $\varepsilon_{22}=6 \%$ (nonlocal multiscale model).

\section{APPENDIX A.}

In the nonlocal DEM-FEM model, the unit cell is constrained by periodic boundary conditions to be compatible with a macroscopic nonlocal strain measure. This nonlocal strain field is obtained by a nonlocal operator. This nonlocal operator integrates a local field $\phi(\mathbf{x})$ over a spherical domain and returns the corresponding nonlocal field $\mathcal{N}(\phi(\mathbf{x}))_{R}$ as a weighted average over a spatial neighborhood domain $V$ as follow,

$$
\mathcal{N}(\phi(\mathbf{x}))_{R}=\int_{V} \alpha^{\prime}(\mathbf{x}, \xi) \phi(\mathbf{x}) d \xi
$$

where $\phi(\mathbf{x})$ is some 'local' field in a domain $V$. The kernel function $\alpha^{\prime}(\mathbf{x}, \xi)$ is defined as follows:

$$
\alpha^{\prime}(\mathbf{x}, \xi)=\frac{\alpha(|\mathbf{x}-\xi|)}{\int_{V} \alpha(|\mathbf{x}-\xi|) d \xi}
$$

In Equation (A2), $\alpha(\mathbf{r})$ is a monotonically decreasing nonnegative function of the distance $r=|\mathbf{x}-\xi|$ typically described by Gauss-shaped or Bell-shaped function. In the present model, the Bell-shaped weight function is employed because of its simplicity as follows:

$$
\alpha(r)= \begin{cases}\left(1-\frac{r^{2}}{R^{2}}\right)^{2} & \text { if } 0 \leqslant r \leqslant R \\ 0 & \text { if } r \geq R\end{cases}
$$

where $R$ denotes the interaction radius, which is an intrinsic material parameter measured in experiments or calibrated from micro-structural simulations.

For convenience, it is convenient to exploit the information at the element quadrature point, and thus, approximate Equations (A.1) and (A.2) are given by the following: 


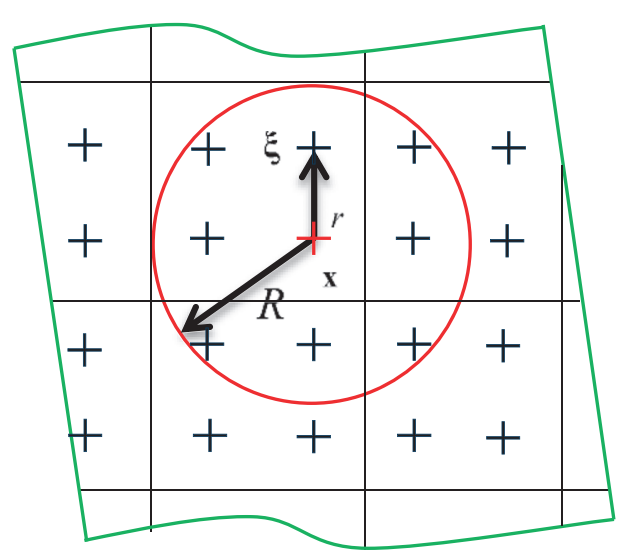

Figure A.1. The spatial neighborhood of the gauss point $\mathbf{x}$ with a characteristic radius $R$.

$$
\begin{aligned}
& \mathcal{N}\left(\phi\left(\mathbf{x}_{I}\right)\right)_{R}=\sum_{\xi_{J} \in Q_{I}} \alpha^{*}\left(\mathbf{x}_{I}, \xi_{J}\right) \phi\left(\xi_{J}\right) \\
& \alpha^{*}\left(\mathbf{x}_{I}, \xi_{J}\right)=\frac{\alpha\left(\mathbf{x}_{I}, \xi_{J}\right)}{\sum_{\xi_{J} \in Q_{I}} \alpha\left(\mathbf{x}_{I}, \xi_{J}\right)}
\end{aligned}
$$

where $\mathbf{x}_{I}$ denotes the position of the quadrature point $I ; Q_{I}$ is a set of quadrature points $\xi_{J} \in Q_{I}$ adjacent to point $\mathbf{x}_{I}$ as shown in Figure A.1 that

$$
\left\{Q_{I}|| \mathbf{x}_{I}-\xi_{J} \mid \leqslant R, \forall \xi_{J} \in Q_{I}\right\}
$$

where the $Q_{I}$ adjacency information for each quadrature point is precomputed in the preprocessing stage. In the staggered nonlocal algorithm [35], the staggered nonlocal operator $\left\langle\phi\left(\mathbf{x}_{I}\right)\right\rangle_{R}$ is defined as follows:

$$
\begin{gathered}
\left\langle\phi\left(\mathbf{x}_{I}\right)\right\rangle_{R}=\sum_{\xi_{J} \in Q_{I}} \alpha^{*}\left(\mathbf{x}_{I}, \xi_{J}\right) \phi^{*}\left(\xi_{J}\right) \\
\phi^{*}\left(\xi_{J}\right)= \begin{cases}\phi_{n+1}\left(\xi_{I}\right) & \text { if } \xi_{J}=\mathbf{x}_{I} \\
\phi_{n+\alpha}\left(\xi_{J}\right) & \text { if } \xi_{J} \neq \mathbf{x}_{I}\end{cases}
\end{gathered}
$$

where the subscript denotes the time step count, $\phi_{n+\alpha}$ denotes the local field computed on the fly, that is, $\alpha$ is either 0 or 1 , which represents either previous or current time step, and $\phi_{n+1}$ is a local field at the current time step $n+1$.

\section{APPENDIX B.}

The numerical implementation of the co-rotational DEM-FEM scheme for large deformation problems is as follows: 
Box B1: Stress updating procedure for coarse-scale corotational framework

Step 1. Compute the coarse-scale deformation gradients at step $n+1 / 2$ and $n+1$

$$
\begin{aligned}
& \mathbf{F}_{n+1}^{c}=\frac{\partial\left(\mathbf{X}+\mathbf{u}_{n+1}^{c}\right)}{\partial \mathbf{X}} ; \\
& \mathbf{F}_{n+1 / 2}^{c}=\frac{\partial\left(\mathbf{X}+\mathbf{u}_{n+1 / 2}^{c}\right)}{\partial \mathbf{X}}
\end{aligned}
$$

Step 2. Compute polar decompositions at step $n+1 / 2$ and $n+1$

$$
\begin{aligned}
& \mathbf{F}_{n+1}^{c}=\mathfrak{R}_{n+1}^{c} \cdot \mathbf{U}_{n+1}^{c} \\
& \mathbf{F}_{n+1 / 2}^{c}=\mathfrak{R}_{n+1 / 2}^{c} \cdot \mathbf{U}_{n+1 / 2}^{c}
\end{aligned}
$$

Step 3. Compute the coarse-scale strain increment over the step from the $\mathbf{B}$ matrix for the coarse-scale finite element

$$
\begin{aligned}
& \Delta \tilde{\boldsymbol{\varepsilon}}_{n+1 / 2}^{c}=\mathbf{B}_{n+1 / 2}\left(\mathbf{u}_{n+1}^{c}-\mathbf{u}_{n}^{c}\right) \\
& \Delta \boldsymbol{\varepsilon}_{n+1 / 2}^{c} \leftarrow \Delta \tilde{\boldsymbol{\varepsilon}}_{n+1 / 2}^{c} \text { (convert } 6 \times 1 \text { vector to symmetric tensor) }
\end{aligned}
$$

Step 4. Apply the nonlocal operator $\langle\cdot\rangle_{R}$ on the coarse-scale strain increment $\Delta \boldsymbol{\varepsilon}_{n+1 / 2}^{c}$ yields the nonlocal strain increment $\left\langle\Delta \boldsymbol{\varepsilon}_{n+1 / 2}^{c}\right\rangle_{R}$, and then rotate the nonlocal strain increment to the corotational frame

$$
\left\langle\Delta \boldsymbol{\varepsilon}_{n+1 / 2}^{\Re}\right\rangle_{R}=\left(\mathfrak{R}_{n+1 / 2}^{c}\right)^{T} \cdot\left\langle\Delta \boldsymbol{\varepsilon}_{n+1 / 2}^{c}\right\rangle_{R} \cdot\left(\mathfrak{R}_{n+1 / 2}^{c}\right)
$$

Invoke the DEM to solve for the coarse-scale corotational Cauchy stress at $n+1$

$\boldsymbol{\sigma}_{n+1}^{\Re} \leftarrow \mathrm{DEM} \leftarrow\left\langle\Delta \boldsymbol{\varepsilon}_{n+1 / 2}^{\Re}\right\rangle_{R}$

Step 5. The coarse-scale corotational Cauchy stress is rotated back to the global Cartesian coordinate system to get the coarse-scale Cauchy stress at step $n+1$ $\boldsymbol{\sigma}_{n+1}^{c}=\left(\mathfrak{R}_{n+1}\right) \cdot \boldsymbol{\sigma}_{n+1}^{\Re} \cdot\left(\mathfrak{R}_{n+1}\right)^{T}$

\section{ACKNOWLEDGEMENTS}

This research is supported by the Earth Materials and Processes program at the US Army Research Office under grant contract W911NF-14-1-0658 and W911NF-15-1-0581, as well as the Mechanics of Material program at National Science Foundation under grant contract CMMI-1462760. These supports are gratefully acknowledged. We thank the reviewers for their constructive suggestion and feedback.

\section{REFERENCES}

1. de Borst R. A generalisation of J2-flow theory for polar continua. Computer Methods in Applied Mechanics and Engineering 1993; 103(3):347-362.

2. Vermeer PA, de Borst R. Non-associated plasticity for soils, concrete and rock. Heron 1984; 29(3):1-64.

3. Kingston M, Spencer A. General yield conditions in plane deformations of granular media. Journal of the Mechanics and Physics of Solids 1970; 18(3):233-243. 
4. Pestana JM, Whittle AJ. Formulation of a unified constitutive model for clays and sands. International Journal for Numerical and Analytical Methods in Geomechanics 1999; 23(12):1215-1243.

5. Pestana JM, Whittle AJ, Salvati LA. Evaluation of a constitutive model for clays and sands: Part I-sand behaviour. International Journal for Numerical and Analytical Methods in Geomechanics 2002; 26(11):1097-1121.

6. Assimaki D, Kausel E, Whittle A. Model for dynamic shear modulus and damping for granular soils. Journal of Geotechnical and Geoenvironmental Engineering 2000; 126(10):859-869.

7. Wang ZL, Dafalias YF, Shen CK. Bounding surface hypoplasticity model for sand. Journal of engineering mechanics 1990; 116(5):983-1001.

8. Manzari MT, Dafalias YF. A critical state two-surface plasticity model for sands. Geotechnique 1997; 47(2):255-272.

9. Lade PV, Duncan JM. Elastoplastic stress-strain theory for cohesionless soil. Journal of the Geotechnical Engineering Division 1975; 101(10):1037-1053.

10. Drucker DC, Prager W. Soil mechanics and plastic analysis or limit design. Quarterly of Applied Mathematics 1952; 10(2): $157-165$.

11. Rudnicki JW, Rice J. Conditions for the localization of deformation in pressure-sensitive dilatant materials. Journal of the Mechanics and Physics of Solids 1975; 23(6):371-394.

12. de Borst R, Sluys LJ, Muhlhuas H-B, Pamin J. Fundamental issues in finite element analyses of localization of deformation. Engineering Computations 1993; 10(2):99-121.

13. Rechenmacher A, Abedi S, Chupin O. Evolution of force chains in shear bands in sands. Geotechnique 2010; 60(5):343-351.

14. Cundall PA. A computer model for simulating progressive large scale movements in blocky rock systems. Proc. Symp. Rock Fracture (ISRM): Nancy, 2013; 2-8.

15. Cundall PA, Strack OD. A discrete numerical model for granular assemblies. Geotechnique 1979; 9(1):47-65.

16. Kouznetsova V, Geers MG, Brekelmans WM. Multi-scale constitutive modelling of heterogeneous materials with a gradient-enhanced computational homogenization scheme. International Journal for Numerical Methods in Engineering 2002; 54(8):1235-1260.

17. Geers M, Kouznetsova V, Brekelmans W. Multi-scale computational homogenization: trends and challenges. Journal of computational and applied mathematics 2010; 234(7):2175-2182.

18. Wellmann C, Lillie C, Wriggers P. Homogenization of granular material modeled by a three-dimensional discrete element method. Computers and Geotechnics 2008; 35(3):394-405.

19. Bardet J., Proubet J. A numerical investigation of the structure of persistent shear bands in granular media. Geotechnique 1991; 41(4):599-613.

20. Wellmann C, Wriggers P. A two-scale model of granular materials. Computer Methods in Applied Mechanics and Engineering 2012; 205:46-58.

21. Li X, Wan K. A bridging scale method for granular materials with discrete particle assembly-Cosserat continuum modeling. Computers and Geotechnics 2011; 38(8):1052-1068.

22. Regueiro RA, Yan B. Concurrent multiscale computational modeling for dense dry granular materials interfacing deformable solid bodies. Bifurcations, Instabilities and Degradations in Geomaterials, Springer, 2011; 251-273.

23. Miehe C, Dettmar J. A framework for micro-macro transitions in periodic particle aggregates of granular materials. Computer Methods in Applied Mechanics and Engineering 2004; 193(3):225-256.

24. Miehe C, Dettmar J, Zäh D. Homogenization and two-scale simulations of granular materials for different microstructural constraints. International Journal for Numerical Methods in Engineering 2010; 83(8 - 9):1206-1236.

25. Stránský J, Jirásek M. Open Source FEM-DEM Coupling. Proc. 18th Int. Conf. Engineering Mechanics, Pragues, 2012;1237-1251.

26. Nguyen TK, Combe G, Gaillerie D, Desrues J. FEM $\times$ DEM modelling of cohesive granular materials: numerical homogenisation and multi-scale simulations. Acta Geophysica 2014; 62(5):1109-1126.

27. Guo N, Zhao J. A coupled FEM/DEM approach for hierarchical multiscale modelling of granular media. International Journal for Numerical Methods in Engineering 2014; 99(11):789-818.

28. Andrade JE, Tu X. Multiscale framework for behavior prediction in granular media. Mechanics of Materials 2009; 41(6):652-669.

29. Sun WC, Kuhn MR, Rudnicki JW. A Micromechanical analysis on permeability evolutions of a dilatant shear band. Proceedings of the 48th American Rock Mechanics Association Symposium, University of Minnesota, 2014.

30. Fish J. Practical Multiscaling. Wiley: Chichester, UK, 2013.

31. Chen W, Fish J. A generalized space-time mathematical homogenization theory for bridging atomistic and continuum scales. International Journal for Numerical Methods in Engineering 2006; 67(2):253-271.

32. Chen W, Fish J. A mathematical homogenization perspective of virial stress. International journal for numerical methods in engineering 2006; 67(2):189-207.

33. Fish J, Chen W, Li R. Generalized mathematical homogenization of atomistic media at finite temperatures in three dimensions. Computer Methods in Applied Mechanics and Engineering 2007; 196(4):908-922.

34. Borja RI, Aydin A. Computational modeling of deformation bands in granular media. I. Geological and mathematical framework. Computer Methods in Applied Mechanics and Engineering 2004; 193(27):2667-2698.

35. Fish J, Jiang T, Yuan Z. A staggered nonlocal multiscale model for a heterogeneous medium. International Journal for Numerical Methods in Engineering 2012; 91(2):142-157.

36. Liu Y, Filonova V, Hu N, Yuan Z, Fish J, Yuan Z, Belytschko T, A regularized phenomenological multiscale damage model. International Journal for Numerical Methods in Engineering 2014; 99(12):867-887. 


\section{A NONLOCAL MULTISCALE DISCRETE-CONTINUUM MODEL FOR GRANULAR MATERIALS}

37. Christoffersen J, Mehrabadi M, Nemat-Nasser S. A micromechanical description of granular material behavior. Journal of Applied Mechanics 1981; 48(2):339-344.

38. Rothenburg L, Selvadurai A. A Micromechanical Definition of the $\{C\}$ auchy Stress Tensor for Particulate Media, 1981.

39. Bagi K. Stress and strain in granular assemblies. Mechanics of materials 1996; 22(3):165-177.

40. Newland P, Allely B. Volume changes in drained taixial tests on granular materials. Geotechnique 1957; 7(1):17-34.

41. Oda M, Iwashita K. Study on couple stress and shear band development in granular media based on numerical simulation analyses. International Journal of Engineering Science 2000; 38(15):1713-1740.

42. Mühlhaus H, Vardoulakis I. The thickness of shear bands in granular materials. Geotechnique 1987; 37(3):271-283.

43. Brown C, Evans R. On the application of couple-stress theories to granular media. Geotechnique 1972; 22(2):356-361.

44. Fish J, Filonova V, Kuznetsov S. Micro-inertia effects in nonlinear heterogeneous media. International Journal for Numerical Methods in Engineering 2012; 91(13):1406-1426.

45. Mühlhaus H., Oka F. Dispersion and wave propagation in discrete and continuous models for granular materials. International Journal of Solids and Structures 1996; 33(19):2841-2858.

46. Belytschko T, Liu WK, Moran B, Elkhodary K. Nonlinear Finite Elements for Continua and Structures. John Wiley \& Sons, Chichester, 2013.

47. Bardet J, Proubet J. Adaptative dynamic relaxation for statics of granular materials. Computers \& Structures 1991; 39(3):221-229.

48. Sun W, Kuhn MR, Rudnicki JW. A multiscale DEM-LBM analysis on permeability evolutions inside a dilatant shear band. Acta Geotechnica 2013; 8(5):465-480.

49. Suzuki K, Kuhn MR. Uniqueness of discrete element simulations in monotonic biaxial shear tests. International Journal of Geomechanics 2013; 14(5):06014010.

50. Tu X, Andrade JE. Criteria for static equilibrium in particulate mechanics computations. International Journal for Numerical Methods in Engineering 2008; 75(13):1581-1606.

51. Padbidri JM, Mesarovic SD. Acceleration of DEM algorithm for quasistatic processes. International Journal for Numerical Methods in Engineering 2011; 86(7):816-828.

52. Ng TT. Input parameters of discrete element methods. Journal of Engineering Mechanics 2006; 132(7):723-729.

53. Filonova V, Liu Y, Bailakanavar M, Fish J, Yuan Z. Corotational formulation of reduced order homogenization. CMC: Computers, Materials \& Continua 2013; 34(3):177-198.

54. Yuan Z, Fish J. Nonlinear multiphysics finite element code architecture in object oriented Fortran environment. Finite Elements in Analysis and Design 2015; 99:1-15.

55. Kuhn MR. OVAL and OVALPLOT: Programs for analyzing dense particle assemblies with the discrete element method, 2006. (Available from: http//www.egr.up.edu/contrib/kuhn/oval.) [Accessed on 14 October 2009].

56. Onate E, Rojek J. Combination of discrete element and finite element methods for dynamic analysis of geomechanics problems. Computer Methods in Applied Mechanics and Engineering 2004; 193(27):3087-3128.

57. Kuhn MR. Structured deformation in granular materials. Mechanics of Materials 1999; 31(6):407-429.

58. Cundall P. Distinct element models of rock and soil structure. Analytical and Computational Methods in Engineering Rock Mechanics 1987; 4:129-163.

59. Thornton C. Numerical simulations of deviatoric shear deformation of granular media. Géotechnique 2000; 50(1):43-53.

60. Lin X, Ng TT. A three-dimensional discrete element model using arrays of ellipsoids. Geotechnique 1997; 47(2):319-329.

61. Makse HA, Gland N, Johnson DL, Schwartz LM. Why effective medium theory fails in granular materials. Physical Review Letters 1999; 83(24):5070-5073.

62. Hill R. Elastic properties of reinforced solids: some theoretical principles. Journal of the Mechanics and Physics of Solids 1963; 11(5):357-372.

63. Ostoja-Starzewski M. Material spatial randomness: from statistical to representative volume element. Probabilistic Engineering Mechanics 2006; 21(2):112-132.

64. Ostoja-Starzewski M, Du X, Khisaeva ZF, Li W. Comparisons of the size of the representative volume element in elastic, plastic, thermoelastic, and permeable random microstructures. International Journal for Multiscale Computational Engineering 2007; 5(2):73-82.

65. Sun W, Andrade JE, Rudnicki JW. Multiscale method for characterization of porous microstructures and their impact on macroscopic effective permeability. International Journal for Numerical Methods in Engineering 2011; 88(12): $1260-1279$.

66. Meier H, Steinmann P, Kuhl E. Towards multiscale computation of confined granular media-Contact forces, stresses and tangent operators. Technische Mechanik 2008; 28(1):32-42.

67. Belytschko T, Loehnert S, Song JH. Multiscale aggregating discontinuities: a method for circumventing loss of material stability. International Journal for Numerical Methods in Engineering 2008; 73(6):869-894.

68. Belytschko T, Bindeman LP. Assumed strain stabilization of the eight-node hexahedral element. Computer Methods in Applied Mechanics and Engineering 1993; 105(2):225-260.

69. Sun W, Ostien JT, Salinger AG. A stabilized assumed deformation gradient finite element formulation for strongly coupled poromechanical simulations at finite strain. International Journal for Numerical and Analytical Methods in Geomechanics 2013; 37(16):2755-2788. 


\section{Y. LIU ET AL.}

70. Bao H, Bielak J, Ghattas O, Kallivokas LF. Large-scale simulation of elastic wave propagation in heterogeneous media on parallel computers. Computer Methods in Applied Mechanics and Engineering 1998; 152(1):85-102.

71. Zeghal M, Elgamal AW. Site response and vertical seismic arrays. Progress in Structural Engineering and Materials 2000; 2(1):92-101.

72. Zeghal M, Oskay C. Local system identification analyses of the dynamic response of soil systems. Soil Dynamics and Earthquake Engineering 2002; 22(9):985-993.

73. Borja RI, Sun WC. Estimating inelastic sediment deformation from local site response simulations. Acta Geotechnica 2007; 2(3):183-195.

74. Borja RI, and Sun W. Coseismic sediment deformation during the 1989 Loma Prieta earthquake. Journal of Geophysical Research: Solid Earth (1978-2012) 2008; 113:B08314. DOI: 10.1029/2007JB005265.

75. Bielak J, Christiano P. On the effective seismic input for non-linear soil-structure interaction systems. Earthquake Engineering \& Structural Dynamics 1984; 12(1):107-119.

76. Yoshimura C, Bielak J, Hisada Y, Fernandez A. Domain reduction method for three-dimensional earthquake modeling in localized regions, part II: Verification and applications. Bulletin of the Seismological Society of America 2003; 93(2):825-841.

77. Prevost JH. A simple plasticity theory for frictional cohesionless soils. International Journal of Soil Dynamics and Earthquake Engineering 1985; 4(1):9-17.

78. Zamani N, El Shamy U. Analysis of wave propagation in dry granular soils using DEM simulations. Acta Geotechnica $2011 ; 6(3): 167-182$.

79. Sadd MH, Adhikari G, Cardoso F. DEM simulation of wave propagation in granular materials. Powder Technology 2000; 109(1-3):222-233.

80. Sadd MH, Tai Q, Shukla A. Contact law effects on wave propagation in particulate materials using distinct element modeling. International Journal of Non-Linear Mechanics 1993; 28(2):251-265.

81. Zeghal M. Discrete-element method investigation of the resilient behavior of granular materials. Journal of Transportation Engineering 2004; 130(4):503-509.

82. Wang C, Tannant D, Lilly P. Numerical analysis of the stability of heavily jointed rock slopes using PFC2D. International Journal of Rock Mechanics and Mining Sciences 2003; 40(3):415-424.

83. Casagrande A. Characteristics of cohensionless soils affecting the stability of slops and earth fills. Boston Contributions to Soil Mechanics 1925-1940, 1940. Boston Society of Civil Engineers, 257-276.

84. Bazant ZP. and Jirásek,M. Nonlocal integral formulations of plasticity and damage: survey of progress. Journal of Engineering Mechanics 2002; 128(11):1119-1149.

85. Sun W, Mota A. A multiscale overlapped coupling formulation for large-deformation strain localization. Computational Mechanics 2014:1-18.

86. Fleck N, Hutchinson J. A reformulation of strain gradient plasticity. Journal of the Mechanics and Physics of Solids 2001; 49(10):2245-2271.

87. Belytschko T, Fish J, Engelmann BE. A finite element with embedded localization zones. Computer Methods in Applied Mechanics and Engineering 1988; 70(1):59-89.

88. Borja RI. A finite element model for strain localization analysis of strongly discontinuous fields based on standard Galerkin approximation. Computer Methods in Applied Mechanics and Engineering 2000; 190(11):1529-1549.

89. Borja RI. Finite element simulation of strain localization with large deformation: capturing strong discontinuity using a Petrov-Galerkin multiscale formulation. Computer Methods in Applied Mechanics and Engineering 2002; 191(27):2949-2978.

90. Dolbow J, Belytschko T. A finite element method for crack growth without remeshing. International Journal for Numerical Methods in Engineering 1999; 46(1):131-150.

91. Yang Q, Mota A, Ortiz M. A class of variational strain-localization finite elements. International Journal for Numerical Methods in Engineering 2005; 62(8):1013-1037.

92. Holtzman R, Silin DB, Patzek TW. Mechanical properties of granular materials: a variational approach to grain-scale simulations. International Journal for Numerical and Analytical Methods in Geomechanics 2009; 33(3):391-404. 